\title{
Nonlinear transverse cascade and two-dimensional magnetohydrodynamic subcritical turbulence in plane shear flows
}

\author{
G. R. Mamatsashvili* \\ Department of Physics, Faculty of Exact and Natural Sciences, Tbilisi State University, Tbilisi 0179, Georgia \\ D. Z. Gogichaishvili \\ Department of Physics, The University of Texas at Austin, Austin, Texas 78712, USA \\ G. D. Chagelishvili \\ Institute of Geophysics, Tbilisi State University, Tbilisi 0193, Georgia \\ and Abastumani Astrophysical Observatory, Ilia State University, Tbilisi 0162, Georgia \\ W. Horton \\ Institute for Fusion Studies, The University of Texas at Austin, Austin, Texas 78712, USA \\ (Received 2 December 2013; published 2 April 2014)
}

\begin{abstract}
We find and investigate via numerical simulations self-sustained two-dimensional turbulence in a magnetohydrodynamic flow with a maximally simple configuration: plane, noninflectional (with a constant shear of velocity), and threaded by a parallel uniform background magnetic field. This flow is spectrally stable, so the turbulence is subcritical by nature and hence it can be energetically supported just by a transient growth mechanism due to shear flow non-normality. This mechanism appears to be essentially anisotropic in the spectral (wave-number) plane and operates mainly for spatial Fourier harmonics with streamwise wave numbers less than the ratio of flow shear to Alfvén speed, $k_{y}<S / u_{A}$ (i.e., the Alfvén frequency is lower than the shear rate). We focus on analysis of the character of nonlinear processes and the underlying self-sustaining scheme of the turbulence, i.e., on the interplay between linear transient growth and nonlinear processes, in the spectral plane. Our study, being concerned with a new type of energy-injecting process for turbulence-the transient growth-represents an alternative to the main trends of magnetohydrodynamic (MHD) turbulence research. We find similarity of the nonlinear dynamics to the related dynamics in hydrodynamic flows: to the bypass concept of subcritical turbulence. The essence of the analyzed nonlinear MHD processes appears to be a transverse redistribution of kinetic and magnetic spectral energies in the wave-number plane [as occurs in the related hydrodynamic flow; see Horton et al., Phys. Rev. E 81, 066304 (2010)] and differs fundamentally from the existing concepts of (anisotropic direct and inverse) cascade processes in MHD shear flows.
\end{abstract}

DOI: 10.1103/PhysRevE.89.043101

\section{INTRODUCTION}

The problem of the onset and self-sustenance of turbulence in spectrally stable nonuniform flows is a challenge to fluid dynamics research. The efforts in this direction significantly increased in the 1990s with the understanding and rigorous description of the non-normal nature of nonuniform, or shear, flows (see, e.g., Refs. [1-5]) and its direct consequences, such as the possibility of finite-time, or transient, growth of perturbations in spectrally stable shear flows (e.g., Refs. [6-9]). Classical (direct and inverse) nonlinear cascade processes, even if anisotropic, are in fact unable to provide self-sustenance of perturbations (turbulence) when transiently (nonexponentially) growing modes are present in the flow. In the case of a specific shear flow, however, turbulence can self-organize and be self-sustained through the subtle interplay of the linear transient and nonlinear processes, where the flow shear acts, through the Reynolds stress, to continuously supply the turbulence with energy, thanks to an essential constructive feedback provided by the nonlinear processes [10-15].

\footnotetext{
*george.mamatsashvili@tsu.ge
}

PACS number(s): 95.30.Qd, 47.20.-k, 47.27.-i, 52.30.-q

The direct (nonlinear) cascade - a central process in Kolmogorov's phenomenology-is a consequence of the existence of the so-called inertial range in spectral (Fourier, or wave-number) space, which is free of the action of linear energy-exchange processes and, in fact, occupied by nonlinear transfers. Kolmogorov's classical theory of forced turbulence in hydrodynamics (HD) is the following: large-scale (longwavelength) perturbations imposed on the flow are transferred by a direct nonlinear cascade through the inertial range, to short wavelengths, and, ultimately, to the dissipation region. So, the direct cascade, together with linear instability and dissipative phenomena, constitute the well-known scheme of forced turbulence in HD. However, in spectrally stable shear flows, where transient growth of perturbations is the only possibility, the balance of processes leading to the self-sustenance of turbulence should be completely different. The shear-induced transient growth mainly depends on the orientation (and, to a lesser degree, on the value) of the perturbation wave vector: the spatial Fourier harmonics (SFHs) of perturbations having a certain orientation of the wave vector with respect to the shear flow, can draw flow energy and get amplified, whereas harmonics having another orientation of the wave vector give energy back to the flow and decay. In other words, the linear energy-exchange processes are strongly anisotropic in 
wave-number $\mathbf{k}$ space and occur over a broad range of wave numbers without leaving a free room (i.e., inertial range) for the action of nonlinear processes only. This might render Kolmogorov's phenomenology inapplicable to spectrally stable shear flows. A strong anisotropy of the linear processes in shear flows, in turn, leads to anisotropy of nonlinear processes in $\mathbf{k}$ space. In this case, as revealed in Ref. [16], even in the simplest HD shear flow with linear shear, the dominant nonlinear process turns out to be not a direct, but a transverse cascade, that is, a transverse (angular) redistribution of perturbation harmonics over different quadrants of the wave-number plane (e.g., from quadrants where $k_{x} k_{y}>0$ to quadrants where $k_{x} k_{y}<0$, or vice versa). The interplay of this nonlinear redistribution with linear phenomena (transient growth) becomes intricate: it can provide either positive or negative feedback. In the case of positive feedback, the nonlinearity repopulates transiently growing modes and contributes to the self-sustenance of perturbations. This combined action of anisotropic linear and nonlinear processes can, in turn, give rise to an anisotropic energy spectrum, which, in general, is expected to differ from the Kolmogorovian. As a result, the transverse cascade may naturally appear to be a possible keystone of the bypass concept of subcritical turbulence in spectrally stable HD shear flows, which is being actively discussed among the hydrodynamical community (see, e.g., Refs. [13-15,17]).

In this paper, we extend the above study of nonlinear processes in HD flows to magnetohydrodynamic (MHD) flows and investigate subcritical turbulence in the simplest, spectrally stable shear flow of magnetized plasma. We present the results of direct numerical simulations (DNS) in the Fourier plane, demonstrating the dominance of the transverse cascade in MHD shear flows too. Specifically, we consider the dynamics of two-dimensional (2D; with zero spanwise wave number, $k_{z}=0$ ) perturbations in unbounded incompressible MHD fluid flow with a linear shear of velocity threaded by a uniform background magnetic field directed parallel to the flow. This flow configuration is spectrally stable in the linear regime [18,19] and therefore should be dominated by the above-mentioned shear-induced transient phenomena [20]. Our main goals are

(i) to examine the subcritical transition to turbulence and subsequent self-sustaining dynamics by DNS;

(ii) to describe the general behavior of nonlinear processes (transfers) - transverse cascade-in the presence of shear by carrying out an analysis of these processes in the Fourier plane; and

(iii) to show that the nonlinear transverse cascade is a keystone of self-sustaining dynamics of the turbulence in this simple open MHD flow system.

The last point will allow us to find out in what form the bypass concept of subcritical turbulence can be realized in spectrally stable MHD shear flows.

MHD turbulence phenomenon is ubiquitous in nature and is very important in engineering applications. So, it is natural that there is an enormous amount of research devoted to it, starting with seminal papers [21,22] and their extensions [23,24]. To date, the main trends, including cases of forced and freely decaying MHD turbulence as well as MHD turbulence with a background magnetic field, established over decades have been thoroughly analyzed in a number of review articles and books (see, e.g., Refs. [25-27] and references therein). Most of these analyses commonly focus on turbulence dynamics in wave-number space. However, the case of MHD turbulence in smooth shear flows that we study here involves fundamental novelties: an energy-supplying process for turbulence is flow non-normality-induced linear transient growth. The latter anisotropically injects energy into turbulence over a broad range of length scales and, consequently, rules out the inertial range of the sole activity of nonlinearity and leads to a complex interplay of linear and nonlinear processes. These circumstances give rise to new types of processes in turbulence dynamics that are not accounted for in the main trends of MHD turbulence research.

Magnetized shear flows have been considered in a number of papers [28-30]. However, the range of target parameters adopted in these studies excludes transient growth effects due to shear and novelties associated with it. So, these investigations still belong to the existing trends of MHD turbulence research. For instance, these studies consider the limit of a strong background magnetic field, $\mathbf{B}_{\mathbf{0}}$, along the flow, where the Alfvén frequency of modes with wave number $\mathbf{k}, \omega_{A}=\mathbf{k} \cdot \mathbf{B}_{0} /\left(4 \pi \rho_{0}\right)^{1 / 2}$ ( $\rho_{0}$ is the equilibrium density), is higher than the shear rate of the mean flow, and since transient phenomena responsible for energy injection from shear flow into perturbation harmonics are inefficient in this case, external forcing (peaked at certain wave numbers) is included to drive turbulence. In contrast to this, in our case, the magnetic field is weak and the adopted parameters permit an effective transient exchange of energy between the mean flow and the perturbation harmonics; this actually should serve to drive turbulence without any external forcing. In this regard, in Refs. [31-35], the dynamics of MHD turbulence is investigated in a somewhat similar setup: astrophysical (protoplanetary) disk flows with Keplerian shear and an imposed large-scale magnetic field which is typically weak (i.e., usual plasma $\beta \gg$ 1 in disks; see, e.g., Ref. [36]). This means that there exists harmonics whose Alfvén frequency is smaller than the shear parameter, as in our case. However, in Refs. [32,35], although turbulence dynamics is analyzed in Fourier space, the magnetic field is directed perpendicular to the flow, and consequently shear-induced transient phenomena differ from those studied here. On the other hand, Refs. [31,33,34], similarly to our study, consider the orientation for the magnetic field along the mean flow (i.e., azimuthal for disk flows). They observe three-dimensional (3D) self-sustained turbulence, which is expected to be governed by transient processes of a type similar to those of the 2D shear turbulence studied here, but since the turbulence dynamics (energy injection and transfers) was not investigated in spectral space in those studies, identification of shear-induced effects is not straightforward in their analysis.

The Earth's magnetosphere, created by the interaction of the solar wind with the Earth's magnetic field, represents a huge "laboratory" of various MHD turbulences. In different parts of this laboratory (e.g., ion foreshock, magnetosheath, LL magnetopause, polar cusps, ionosphere, magnetotail) characteristic parameters vary greatly from each other. There are shear flows, different orientations of the magnetic field, different values of the plasma $\beta$ parameter, anisotropic magnetic pressure, magnetic reconnection, etc. (see, e.g., Ref. [37] for a recent review). Evidently, it is hard to seek an immediate realization 
of the proposed scheme of MHD shear turbulence in the magnetized environment of the Earth. Still, certain areas can be identified where a similar configuration and course of events are realized. This, first of all, implies high- $\beta$ regions with shear flows and a mean magnetic field parallel to the flow velocity. Generally, such regions are in the magnetotail, magnetosheath, and cusp, but a definite view can be obtained after a detailed investigation of the dynamical processes therein.

The specific nature of nonlinear processes, which we focus on in our study is, in many respects, a consequence of the shearinduced transient linear dynamics described in Refs. [20,3840]. We, particularly, follow a recent paper [40] in which the linear dynamics of pseudo-Alfvén waves (P-AWs) and shearAlfvén waves (S-AWs) is described in a 3D MHD flow with linear shear and a parallel magnetic field. Specifically, it is shown there that:

(1) Counter-propagating P-AWs are coupled to each other, while S-AWs are not coupled to each other, but are asymmetrically coupled to P-AWs; S-AWs do not participate in the linear dynamics of P-AWs.

(2) The linear coupling of counter-propagating waves determines the transient growth (over-reflection).

(3) The transient growth of S-AWs is somewhat smaller compared with that of P-AWs.

(4) Waves with a smaller streamwise wave number, $k_{y}$, exhibit stronger transient growth.

(5) Maximal transient growth (and over-reflection) of the wave energy occurs for 2D waves with $k_{z}=0$.

These preliminary linear results served as a natural starting point of the present study of nonlinear dynamics of 2D perturbations with $k_{z}=0$ and a white-noise initial spectrum in the $\mathbf{k}$ plane using DNS with a spectral code.

The paper is organized as follows. Section II is devoted to the physical model and derivation of dynamical equations in the spectral plane. The DNS of the turbulence dynamics is presented in Sec. III. In Sec. IV, we perform an analysis of the numerical results, focusing on the activity of linear and nonlinear processes in the spectral plane. A summary and discussion are given in Sec. V.

\section{PHYSICAL MODEL AND EQUATIONS}

The motion of an incompressible conducting fluid with constant viscosity, $v$, and ohmic resistivity, $\eta$, is governed by the basic equations of MHD,

$$
\begin{gathered}
\frac{\partial \mathbf{U}}{\partial t}+(\mathbf{U} \cdot \nabla) \mathbf{U}=-\frac{\nabla P}{\rho}+\frac{(\mathbf{B} \cdot \nabla) \mathbf{B}}{4 \pi \rho}+v \nabla^{2} \mathbf{U}, \\
\frac{\partial \mathbf{B}}{\partial t}=\nabla \times(\mathbf{U} \times \mathbf{B})+\eta \nabla^{2} \mathbf{B}, \\
\nabla \cdot \mathbf{U}=0, \\
\nabla \cdot \mathbf{B}=0,
\end{gathered}
$$

where $\rho$ is the fluid density, $\mathbf{U}$ is the velocity, $\mathbf{B}$ is the magnetic field, and $P$ is the total pressure, equal to the sum of the thermal and magnetic pressures.

Equations (1)-(4) have a stationary equilibrium solution: an unbounded plane Couette flow along the $y$ axis with a linear shear of velocity in the the $x$ direction, $\mathbf{U}_{0}=(0,-S x, 0)$, and threaded by a uniform background magnetic field parallel to the flow, $\mathbf{B}_{0}=\left(0, B_{0 y}, 0\right)$. Without loss of generality, the constant shear parameter $S$ and $B_{0 y}$ are chosen to be positive, $S, B_{0 y}>0$. The equilibrium density $\rho_{0}$ and total pressure $P_{0}$ are spatially constant. Such a simple configuration of an unbounded flow with a linear shear of the velocity profile corresponds, for example, to plasma flow in astrophysical accretion disks in the framework of the widely used local shearing box approximation (e.g., Ref. [31]) as well as to flows of magnetized plasma in the laboratory (e.g., Refs. [28,29]). It allows us to grasp key effects of shear on the perturbation dynamics and, ultimately, on the resulting MHD turbulent state in kinematically nonuniform plasma flows.

Consider 2D perturbations of the velocity, total pressure, and magnetic field, $\mathbf{u}, p$, and $\mathbf{b}$, which are independent of the vertical $z$ coordinate $(\partial / \partial z=0)$, about the equilibrium. In this case, the evolution in the horizontal $(x, y)$ plane is decoupled from that of the $z$ components of the perturbed velocity and magnetic field, so we set them to zero: $u_{z}=b_{z}=0$. Representing the total fields as the sum of the equilibrium and perturbed values, $\mathbf{U}=\mathbf{U}_{0}+\mathbf{u}, P=P_{0}+p$, and $\mathbf{B}=\mathbf{B}_{0}+\mathbf{b}$, substituting these into Eqs. (1)-(4), and rearranging the nonlinear terms with the help of Eqs. (3) and (4), we arrive at the following system governing the dynamics of perturbations with arbitrary amplitude:

$$
\begin{gathered}
\left(\frac{\partial}{\partial t}-S x \frac{\partial}{\partial y}\right) u_{x} \\
=-\frac{1}{\rho_{0}} \frac{\partial p}{\partial x}+\frac{B_{0 y}}{4 \pi \rho_{0}} \frac{\partial b_{x}}{\partial y}+v \nabla^{2} u_{x} \\
+\frac{\partial}{\partial y}\left(\frac{b_{x} b_{y}}{4 \pi \rho_{0}}-u_{x} u_{y}\right)+\frac{\partial}{\partial x}\left(\frac{b_{x}^{2}}{4 \pi \rho_{0}}-u_{x}^{2}\right) \\
\left(\frac{\partial}{\partial t}-S x \frac{\partial}{\partial y}\right) u_{y} \\
=S u_{x}-\frac{1}{\rho_{0}} \frac{\partial p}{\partial y}+\frac{B_{0 y}}{4 \pi \rho_{0}} \frac{\partial b_{y}}{\partial y}+v \nabla^{2} u_{y} \\
+\frac{\partial}{\partial x}\left(\frac{b_{x} b_{y}}{4 \pi \rho_{0}}-u_{x} u_{y}\right)+\frac{\partial}{\partial y}\left(\frac{b_{y}^{2}}{4 \pi \rho_{0}}-u_{y}^{2}\right) \\
\left(\frac{\partial}{\partial t}-S x \frac{\partial}{\partial y}\right) b_{x} \\
=-S b_{x}+B_{0 y} \frac{\partial u_{y}}{\partial y}+\eta v_{x}^{2} b_{y}-\frac{\partial}{\partial x}\left(u_{x} b_{y}-u_{y} b_{x}\right) \\
\left(\frac{\partial}{\partial t}\right) b_{y} b_{x}+\frac{\partial}{\partial y}\left(u_{x} b_{y}-u_{y} b_{x}\right) \\
=
\end{gathered}
$$


We solve Eqs. (5)-(10) in a rectangular 2D domain with sizes $L_{x}$ and $L_{y}$, respectively, in the $x$ and $y$ directions, $-L_{x} / 2 \leqslant x \leqslant L_{x} / 2$ and $-L_{y} / 2 \leqslant y \leqslant L_{y} / 2$, divided into $N_{x} \times N_{y}$ cells. Since we consider an unbounded flow with linear shear, we adopt boundary conditions commonly used in similar cases of MHD simulations of astrophysical disk flows in the local shearing box approximation (e.g., Refs. [31$33,35,41])$. Namely, for the perturbations of all quantities, we impose periodic boundary conditions in the $y$ direction and shearing-periodic in the $x$ direction. That is, the $x$ boundaries are initially periodic, but shear with respect to each other as time goes by, becoming, again, periodic at discrete moments $t_{n}=n L_{y} / S L_{x}$, where $n=1,2, \ldots$ is a positive integer. This can be written as

$$
\begin{aligned}
& f(x, y, t)=f\left(x+L_{x}, y-S L_{x} t, t\right) \quad(x \text { boundary }), \\
& f(x, y, t)=f\left(x, y+L_{y}, t\right) \quad(y \text { boundary }),
\end{aligned}
$$

where $f \equiv(\mathbf{u}, p, \mathbf{b})$ denotes any of the perturbed quantities. These boundary conditions ensure natural evolution of shearing plane waves within the domain, as it would be in an unbounded constant shear flow.

\section{A. Energy equation}

In this subsection, we derive dynamical equations for kinetic and magnetic energies in order to gain insight into the interplay of the flow shear and nonlinearity in the selfsustenance of perturbations. The perturbation kinetic and magnetic energies are defined, respectively, as

$$
E_{K}=\frac{\rho_{0} \mathbf{u}^{2}}{2}, \quad E_{M}=\frac{\mathbf{b}^{2}}{8 \pi} .
$$

Using the main Eqs. (5)-(10) and the above shearing box boundary conditions, after some algebra, we can readily derive the evolution equation for the domain-averaged kinetic and magnetic energies,

$$
\begin{aligned}
\frac{d}{d t}\left\langle E_{K}\right\rangle & \\
= & S\left\langle\rho_{0} u_{x} u_{y}\right\rangle+\frac{B_{0 y}}{4 \pi}\left\langle u_{x} \frac{\partial b_{x}}{\partial y}+u_{y} \frac{\partial b_{y}}{\partial y}\right\rangle \\
& +\frac{1}{4 \pi}\left\langle u_{x} b_{y} \frac{\partial b_{x}}{\partial y}+\frac{u_{x}}{2} \frac{\partial b_{x}^{2}}{\partial x}+\frac{u_{y}}{2} \frac{\partial b_{y}^{2}}{\partial y}+u_{y} b_{x} \frac{\partial b_{y}}{\partial x}\right\rangle \\
& -\rho_{0} v\left\langle\left(\nabla u_{x}\right)^{2}+\left(\nabla u_{y}\right)^{2}\right\rangle,
\end{aligned}
$$

$$
\begin{aligned}
& \frac{d}{d t}\left\langle E_{M}\right\rangle \\
& =S\left\langle-\frac{b_{x} b_{y}}{4 \pi}\right\rangle+\frac{B_{0 y}}{4 \pi}\left\langle b_{x} \frac{\partial u_{x}}{\partial y}+b_{y} \frac{\partial u_{y}}{\partial y}\right\rangle \\
& \quad+\frac{1}{4 \pi}\left\langle b_{x} \frac{\partial}{\partial y}\left(u_{x} b_{y}\right)+\frac{b_{x}^{2}}{2} \frac{\partial u_{x}}{\partial x}+\frac{b_{y}^{2}}{2} \frac{\partial u_{y}}{\partial y}+b_{y} \frac{\partial}{\partial x}\left(u_{y} b_{x}\right)\right\rangle \\
& \quad-\frac{\eta}{4 \pi}\left\langle\left(\nabla b_{x}\right)^{2}+\left(\nabla b_{y}\right)^{2}\right\rangle,
\end{aligned}
$$

where the angle brackets denote a spatial average, $\langle\cdots\rangle=$ $\iint \ldots d x d y / L_{x} L_{y}$, with the integral being taken over an entire domain. Adding up Eqs. (11) and (12), the cross terms of linear origin, proportional to $B_{0 y}$, and nonlinear terms cancel out due to the boundary conditions, and we obtain the equation for the total energy $E=E_{K}+E_{M}$,

$$
\begin{aligned}
\frac{d\langle E\rangle}{d t}= & S\left\langle\rho_{0} u_{x} u_{y}-\frac{b_{x} b_{y}}{4 \pi}\right\rangle-\rho_{0} v\left\langle\left(\nabla u_{x}\right)^{2}\right. \\
& \left.+\left(\nabla u_{y}\right)^{2}\right\rangle-\frac{\eta}{4 \pi}\left\langle\left(\nabla b_{x}\right)^{2}+\left(\nabla b_{y}\right)^{2}\right\rangle .
\end{aligned}
$$

The first term on the right-hand side of Eq. (13) is the shear parameter, $S$, multiplied by the total stress in the angle brackets. The total stress is the sum of the Reynolds, $\rho_{0} u_{x} u_{y}$, and Maxwell, $-b_{x} b_{y} / 4 \pi$, stresses, which describe, respectively, the exchange of kinetic and magnetic energies between perturbations and the background flow in Eqs. (11) and (12). Note that they originate from the linear terms proportional to shear on the right hand sides of Eqs. (6) and (8). These stresses also determine the rate of momentum transport (see, e.g., Refs. [29,31,42]) and, thus, are one of the important quantities characterizing shear flow turbulence. The second and third terms describe energy dissipation due to viscosity and resistivity, respectively. Note that the net contribution from nonlinear terms has canceled out in the total energy evolution, Eq. (13), after averaging over the domain. Thus, only Reynolds and Maxwell stresses can supply perturbations with energy, extracting it from the mean flow due to shear; the other two terms are negative definite and dissipative. In the case of shear flow turbulence studied below, these stresses ensure energy injection into turbulent fluctuations. The nonlinear terms, not directly tapping into the shear flow energy and therefore not changing the total perturbation energy, serve only to redistribute energy gained by means of the stresses among Fourier harmonics of perturbations with different wave numbers (see below). In the absence of shear $(S=0)$, the contribution from the Reynolds and Maxwell stresses disappears in Eq. (13) and hence the total perturbation energy cannot grow, gradually decaying due to viscosity and resistivity.

\section{B. Spectral representation of the equations}

Before proceeding further, we normalize the variables by taking the shear time, $S^{-1}$, as the unit of time, the Alfvén speed, $u_{A}=B_{0 y} /\left(4 \pi \rho_{0}\right)^{1 / 2}$, as the unit of velocity, $\ell \equiv u_{A} S^{-1}$ as the unit of length, and $B_{0 y}$ as the unit of the magnetic field perturbations:

$$
\begin{gathered}
S t \rightarrow t, \quad\left(\frac{x}{\ell}, \frac{y}{\ell}\right) \rightarrow(x, y), \quad \frac{\mathbf{u}}{u_{A}} \rightarrow \mathbf{u}, \\
\frac{p}{\rho_{0} u_{A}^{2}} \rightarrow p, \quad \frac{\mathbf{b}}{B_{0 y}} \rightarrow \mathbf{b}, \quad \frac{E_{K, M}}{\rho_{0} u_{A}^{2}} \rightarrow E_{K, M} .
\end{gathered}
$$

Viscosity and resistivity are characterized by hydrodynamic, $\mathrm{Re}$, and magnetic, Rm, Reynolds numbers, defined here, for convenience, in terms of $u_{A}$ and $\ell$ as

$$
\operatorname{Re}=\frac{u_{A} \ell}{v}=\frac{u_{A}^{2}}{v S}, \quad \operatorname{Rm}=\frac{u_{A} \ell}{\eta}=\frac{u_{A}^{2}}{\eta S} .
$$


These numbers are also referred to, respectively, as viscous and resistive Elsasser numbers (e.g., Ref. [35]). The strength of the imposed mean magnetic field is measured by the ratio of the mean flow kinetic energy to the magnetic energy within the domain:

$$
\beta=\frac{\pi \rho_{0} S^{2} L_{x}^{2}}{3 B_{0 y}^{2}}=\frac{S^{2} L_{x}^{2}}{12 u_{A}^{2}}=\frac{L_{x}^{2}}{12 \ell^{2}} .
$$

For further analysis, we need to do a spectral representation of the main equations. We decompose the perturbations into SFHs,

$$
f(\mathbf{r}, t)=\int \bar{f}(\mathbf{k}, t) \exp (\mathrm{ik} \cdot \mathbf{r}) d^{2} \mathbf{k},
$$

where, as before, $f \equiv(\mathbf{u}, p, \mathbf{b})$ denotes the perturbations and $\bar{f} \equiv(\overline{\mathbf{u}}, \bar{p}, \overline{\mathbf{b}})$ is their corresponding Fourier transforms $\left(k_{z}=0\right.$ for $z$-independent 2D perturbations and $d^{2} \mathbf{k} \equiv d k_{x} d k_{y}$ ). Substituting decomposition (14) into Eqs. (5)-(10) and taking into account the above normalization, we arrive at the equations governing the dynamics of perturbation SFHs in the spectral plane,

$$
\begin{gathered}
\left(\frac{\partial}{\partial t}+k_{y} \frac{\partial}{\partial k_{x}}\right) \bar{u}_{x} \\
=-\mathrm{i} k_{x} \bar{p}+\mathrm{i} k_{y} \bar{b}_{x}-\frac{k^{2}}{\operatorname{Re}} \bar{u}_{x}+\mathrm{i} k_{y} N_{1}+\mathrm{i} k_{x} N_{2}, \\
\left(\frac{\partial}{\partial t}+k_{y} \frac{\partial}{\partial k_{x}}\right) \bar{u}_{y} \\
=\bar{u}_{x}-\mathrm{i} k_{y} \bar{p}+\mathrm{i} k_{y} \bar{b}_{y}-\frac{k^{2}}{\operatorname{Re}} \bar{u}_{y}+\mathrm{i} k_{x} N_{1}+\mathrm{i} k_{y} N_{3}, \\
\left(\frac{\partial}{\partial t}+k_{y} \frac{\partial}{\partial k_{x}}\right) \bar{b}_{x}=\mathrm{i} k_{y} \bar{u}_{x}-\frac{k^{2}}{\mathrm{Rm}} \bar{b}_{x}+\mathrm{i} k_{y} N_{4}, \\
\left(\frac{\partial}{\partial t}+k_{y} \frac{\partial}{\partial k_{x}}\right) \bar{b}_{y}=-\bar{b}_{x}+\mathrm{i} k_{y} \bar{u}_{y}-\frac{k^{2}}{\mathrm{Rm}} \bar{b}_{y}-\mathrm{i} k_{x} N_{4}, \\
k_{x} \bar{u}_{x}+k_{y} \bar{u}_{y}=0, \\
k_{x} \bar{b}_{x}+k_{y} \bar{b}_{y}=0,
\end{gathered}
$$

where $k^{2}=k_{x}^{2}+k_{y}^{2}$ (wave numbers are normalized by $\ell^{-1}$ ). These spectral equations contain the linear as well as the nonlinear, $N_{1}(\mathbf{k}, t), N_{2}(\mathbf{k}, t), N_{3}(\mathbf{k}, t)$, and $N_{4}(\mathbf{k}, t)$, terms that are the Fourier transforms of corresponding linear and nonlinear terms in the original Eqs. (5)-(10). The latter are given by

$$
\begin{aligned}
N_{1}(\mathbf{k}, t)= & \int d^{2} \mathbf{k}^{\prime}\left[\bar{b}_{x}\left(\mathbf{k}^{\prime}, t\right) \bar{b}_{y}\left(\mathbf{k}-\mathbf{k}^{\prime}, t\right)\right. \\
& \left.-\bar{u}_{x}\left(\mathbf{k}^{\prime}, t\right) \bar{u}_{y}\left(\mathbf{k}-\mathbf{k}^{\prime}, t\right)\right], \\
N_{2}(\mathbf{k}, t)= & \int d^{2} \mathbf{k}^{\prime}\left[\bar{b}_{x}\left(\mathbf{k}^{\prime}, t\right) \bar{b}_{x}\left(\mathbf{k}-\mathbf{k}^{\prime}, t\right)\right. \\
& \left.-\bar{u}_{x}\left(\mathbf{k}^{\prime}, t\right) \bar{u}_{x}\left(\mathbf{k}-\mathbf{k}^{\prime}, t\right)\right],
\end{aligned}
$$

$$
\begin{aligned}
N_{3}(\mathbf{k}, t)= & \int d^{2} \mathbf{k}^{\prime}\left[\bar{b}_{y}\left(\mathbf{k}^{\prime}, t\right) \bar{b}_{y}\left(\mathbf{k}-\mathbf{k}^{\prime}, t\right)\right. \\
& \left.-\bar{u}_{y}\left(\mathbf{k}^{\prime}, t\right) \bar{u}_{y}\left(\mathbf{k}-\mathbf{k}^{\prime}, t\right)\right], \\
N_{4}(\mathbf{k}, t)= & \int d^{2} \mathbf{k}^{\prime}\left[\bar{u}_{x}\left(\mathbf{k}^{\prime}, t\right) \bar{b}_{y}\left(\mathbf{k}-\mathbf{k}^{\prime}, t\right)\right. \\
& \left.-\bar{u}_{y}\left(\mathbf{k}^{\prime}, t\right) \bar{b}_{x}\left(\mathbf{k}-\mathbf{k}^{\prime}, t\right)\right]
\end{aligned}
$$

and describe nonlinear triad interactions among the velocity and magnetic field components of SFHs with different wave numbers in the Fourier $\mathbf{k}$ plane. Equations (15)-(20), which are the basis for subsequent analysis, involve two free dissipative parameters, $\mathrm{Re}$ and $\mathrm{Rm}$. Since we consider a finite domain in the physical $(x, y)$ plane, the perturbation dynamics also depends on the smallest wave number available in this domain or, equivalently, on its sizes $L_{x}$ and $L_{y}$, which are the other two free parameters of the problem. Given these parameters and specific initial conditions, Eqs. (15)-(20) fully determine the nonlinear dynamics of the considered system in the Fourier plane. These equations form the mathematical basis of our main goal: to investigate the character of nonlinear processes and self-sustaining scheme of the (subcritical) MHD turbulence in the $\mathbf{k}$ plane in this constant shear flow. Since energy spectra and nonlinear transfers relate to energy equations, following Refs. [16,32,35,43-45], below we derive equations governing the evolution of kinetic and magnetic spectral energies.

Multiplying Eqs. (15) and (16) by $\bar{u}_{x}^{*}$ and $\bar{u}_{y}^{*}$, respectively, combining and adding its complex conjugate, we arrive at the equation for the nondimensional kinetic spectral energy $\bar{E}_{K}=\left|\bar{u}_{x}\right|^{2}+\left|\bar{u}_{y}\right|^{2}$,

$$
\frac{\partial \bar{E}_{K}}{\partial t}+\frac{\partial}{\partial k_{x}}\left(k_{y} \bar{E}_{K}\right)=I_{K}+I_{K-M}+D_{K}+N_{K},
$$

where

$$
\begin{aligned}
I_{K} & =\bar{u}_{x} \bar{u}_{y}^{*}+\bar{u}_{x}^{*} \bar{u}_{y}=-\frac{2 k_{x} k_{y}}{k^{2}} \bar{E}_{K}, \quad D_{K}=-\frac{2 k^{2}}{\operatorname{Re}} \bar{E}_{K}, \\
I_{K-M} & =\mathrm{i} k_{y}\left(\bar{u}_{x}^{*} \bar{b}_{x}+\bar{u}_{y}^{*} \bar{b}_{y}-\bar{u}_{x} \bar{b}_{x}^{*}-\bar{u}_{y} \bar{b}_{y}^{*}\right),
\end{aligned}
$$

and the nonlinear kinetic transfer function $N_{K}(\mathbf{k}, t)$ is given by

$$
\begin{aligned}
N_{K}(\mathbf{k}, t)= & \mathrm{i}\left(k_{y} \bar{u}_{x}^{*}+k_{x} \bar{u}_{y}^{*}\right) N_{1}(\mathbf{k}, t) \\
& +\mathrm{i} k_{x} \bar{u}_{x}^{*}\left[N_{2}(\mathbf{k}, t)-N_{3}(\mathbf{k}, t)\right]+\text { c.c. }
\end{aligned}
$$

Similarly, multiplying Eqs. (17) and (18) by $\bar{b}_{x}^{*}$ and $\bar{b}_{y}^{*}$, respectively, combining and adding its complex conjugate, we obtain the evolution equation for the nondimensional magnetic spectral energy $\bar{E}_{M}=\left|\bar{b}_{x}\right|^{2}+\left|\bar{b}_{y}\right|^{2}$,

$$
\frac{\partial \bar{E}_{M}}{\partial t}+\frac{\partial}{\partial k_{x}}\left(k_{y} \bar{E}_{M}\right)=I_{M}+I_{M-K}+D_{M}+N_{M},
$$

where

$$
\begin{gathered}
I_{M}=-\bar{b}_{x} \bar{b}_{y}^{*}-\bar{b}_{x}^{*} \bar{b}_{y}=\frac{2 k_{x} k_{y}}{k^{2}} \bar{E}_{M}, \\
I_{M-K}=-I_{K-M}, \quad D_{M}=-\frac{2 k^{2}}{\mathrm{Rm}} \bar{E}_{M}
\end{gathered}
$$


and the nonlinear magnetic transfer function $N_{M}(\mathbf{k}, t)$ is given by

$$
N_{M}(\mathbf{k}, t)=\mathrm{i}\left(k_{y} \bar{b}_{x}^{*}-k_{x} \bar{b}_{y}^{*}\right) N_{4}(\mathbf{k}, t)+\text { c.c. }
$$

By inspection of Eqs. (21) and (22), one can distinguish five basic processes underlying the dynamics of $\bar{E}_{K}$ and $\bar{E}_{M}$ :

(1) The quantities $k_{y} \bar{E}_{K}$ and $k_{y} \bar{E}_{M}$ in the second terms on the left-hand sides of Eqs. (21) and (22) are, respectively, the fluxes of the kinetic and magnetic spectral energies parallel to the $k_{x}$ axis. These terms are of linear origin, coming from the convective derivative on the left-hand sides of the main Eqs. (5)-(10), and therefore correspond to the advection by the mean flow. In other words, background shear flow makes the spectral energies (Fourier transforms) "drift" in the $\mathbf{k}$ plane, and SFHs with $k_{y}>0$ and $k_{y}<0$ travel, respectively, along and opposite the $k_{x}$ axis at a speed $\left|k_{y}\right|$, whereas SFHs with $k_{y}=0$ are not advected by the flow. Since $\int d^{2} \mathbf{k} \partial\left(k_{y} \bar{E}_{K, M}\right) / \partial k_{x}=0$, this drift only transports SFHs parallel to the $k_{x}$ axis, without changing the total kinetic and magnetic energies.

(2) The first terms on the right-hand sides, $I_{K}$ and $I_{M}$, are associated with shear; i.e., they originate from linear terms proportional to the shear parameter on the right hand sides of Eqs. (6) and (8) and describe energy exchange between the mean flow and individual SFHs. These terms are related to the domain-averaged nondimensional Reynolds and Maxwell stresses entering Eqs. (11) and (12) through

$$
\begin{aligned}
\left\langle u_{x} u_{y}\right\rangle & =\frac{1}{2} \int I_{K}(\mathbf{k}, t) d^{2} \mathbf{k}, \\
\left\langle-b_{x} b_{y}\right\rangle & =\frac{1}{2} \int I_{M}(\mathbf{k}, t) d^{2} \mathbf{k}
\end{aligned}
$$

and therefore serve as a main source of energy for SFHs (with $k_{y} \neq 0$ ), at the expense of which they can undergo amplification. This shear-induced growth of perturbation SFHs is in fact linear by nature and has a transient character due to the drift in the $\mathbf{k}$ plane $[20,38,39,46,47]$. The SFHs, drifting parallel to the $k_{x}$ axis, go through dynamically important regions in the spectral plane, where energy-supplying linear terms, $I_{K}$ and $I_{M}$, and redistributing nonlinear terms, $N_{K}$ and $N_{M}$, are at work from small and intermediate wave numbers almost up to the dissipation region at large wave numbers (see, e.g., Fig. 6). In the case of turbulence studied below, $I_{K}$ and $I_{M}$ describe the injection, respectively, of kinetic and magnetic energies into turbulent fluctuations as a function of the wave numbers (see also Refs. [32,35]).

(3) The second, cross terms on the right-hand sides, $I_{K-M}$ and $I_{M-K}$, describe exchange between kinetic and magnetic spectral energies. They have opposite signs and therefore cancel out in the total energy budget of SFHs [see Eq. (24) below]. These terms are also of linear origin, corresponding to terms proportional to $B_{0 y}$ (linearized magnetic tension and electromotive forces) in Eqs. (5)-(8).

(4) The third terms on the right-hand sides, $D_{K}$ and $D_{M}$, describe the dissipation of kinetic and magnetic energies due to viscosity and resistivity, respectively. Comparing these dissipation terms with the energy-supplying terms $I_{K}$ and $I_{M}$, we see that viscous and resistive dissipation are important at large wave numbers $k \gtrsim k_{D}=\min (\sqrt{\mathrm{Re}}, \sqrt{\mathrm{Rm}})$, where
$k_{D}$ denotes the effective wave number for which dissipation effects start to play a role.

(5) The fourth terms on the right-hand sides, $N_{K}$ and $N_{M}$, describe nonlinear transfers, respectively, of kinetic and magnetic energies among SFHs with different wave numbers in the $\mathbf{k}$ plane. It follows from the definition of $N_{K}$ and $N_{M}$ that their sum integrated over an entire wave-number plane is equal to zero,

$$
\int\left[N_{K}(\mathbf{k}, t)+N_{M}(\mathbf{k}, t)\right] d^{2} \mathbf{k}=0
$$

which is, in fact, a direct consequence of the vanishing of the nonlinear terms in the total energy, Eq. (13), in the real plane. This implies that the main effect of nonlinearity is only to redistribute (scatter) energy drawn from the mean flow among kinetic and magnetic components of perturbation SFHs with different wave numbers, while leaving the total (kinetic plus magnetic) spectral energy summed over all wave numbers unchanged. In general, nonlinear transfer functions, $N_{K}$ and $N_{M}$, play a central role in MHD turbulence theory: they determine cascades of spectral energies in $\mathbf{k}$ space, leading to the development of their specific spectra. These transfer functions are one of the main focuses of the present analysis. We aim to explore how they operate in the presence of shear, adopting the approach of Refs. [16,43], which numerically studied the nonlinear dynamics of $2 \mathrm{D}$ perturbations in an HD Couette flow by performing a full 2D Fourier analysis of individual terms in the evolution equation for spectral energy, thus allowing for anisotropy of spectra and cascades. In particular, we show below that, like that in the HD shear flow, nonlinear transfers in the quasisteady MHD shear turbulence result in the redistribution of spectral energy among wave-vector angles in the $\mathbf{k}$ plane, which we refer to as a nonlinear transverse cascade, in contrast to classical HD or MHD turbulence without background shear flow, where energy cascade processes change only the wave-vector magnitude, $k=|\mathbf{k}|$, of SFHs (see, e.g., Ref. [25]).

Combining Eqs. (21) and (22), we obtain the equation for the total spectral energy $\bar{E}=\bar{E}_{K}+\bar{E}_{M}$ :

$$
\frac{\partial \bar{E}}{\partial t}+\frac{\partial}{\partial k_{x}}\left(k_{y} \bar{E}\right)=I_{K}+I_{M}+D_{K}+D_{M}+N_{K}+N_{M} .
$$

As mentioned above, the linear cross terms responsible for kinetic and magnetic energy exchange are absent in this equation. The net effect of the nonlinear terms in the total spectral energy budget over all wave numbers is zero according to Eq. (23). Thus, as follows from Eq. (24), the only source for the total perturbation energy is the integral over an entire spectral plane $\int\left(I_{K}+I_{M}\right) d^{2} \mathbf{k}$ that extracts energy from a vast reservoir of shear flow and injects it into perturbations. Since the terms $I_{K}$ and $I_{M}$, as noted above, are of linear origin, the energy extraction and perturbation growth mechanisms are essentially linear by nature. The role of nonlinearity is to continually provide, or regenerate those SFHs in the $\mathbf{k}$ plane that are able to undergo transient growth, drawing on the mean flow energy, and in this way feed the nonlinear state over long times. This scenario of a self-sustained state, based on a 
subtle cooperation between linear and nonlinear processes, is a keystone of the bypass concept of subcritical turbulence in spectrally stable shear flows [10,12-15,17].

\section{NONLINEAR EVOLUTION}

We now turn to an analysis of the nonlinear evolution of perturbations employing modern numerical methods. The main emphasis is on the spectral aspect of the dynamics using the mathematical formalism outlined in the previous section. We start a fiducial run by imposing solenoidal random noise perturbations of the velocity and magnetic field with spatially uniform rms amplitudes $\left\langle\mathbf{u}^{2}\right\rangle^{1 / 2}=\left\langle\mathbf{b}^{2}\right\rangle^{1 / 2}=0.84$ on top of the equilibrium. The computational domain is a square of size $L_{x} \times L_{y}=400 \times 400$ and resolution $N_{x} \times N_{y}=512 \times 512$. The reason for taking a large domain is to encompass wave numbers as small as possible at which, as shown below, the effective transient amplification of SFHs and most of the dynamical activity take place. The minimum and maximum wave numbers of the domain are $k_{x, \min }=k_{y, \min }=2 \pi / L_{x}=$ 0.016 and $k_{x, \max }=k_{y, \max }=\pi N_{x} / L_{x}=4.02$. The viscous and resistive Reynolds numbers are fixed to the values $\mathrm{Re}=$ $\mathrm{Rm}=5$ (corresponding to the magnetic Prandtl number of unity $\operatorname{Pr}=\operatorname{Rm} / \operatorname{Re}=1$ ), so that the dissipation wave number, $k_{D}$, falls in this range, $k_{D}=\sqrt{\mathrm{Re}}=2.24<k_{x \text {, max }}$ [48]. Note also that for the domain size $L_{x}=400$ the above-defined parameter $\beta=L_{x}^{2} / 12=1.33 \times 10^{4}$ is quite large, indicating that the background magnetic field energy is low compared to the kinetic energy of the mean flow and therefore the flow can be regarded as weakly magnetized.

The subsequent time evolution with these initial conditions was followed to $t_{f}=600$ (i.e., for a total of 600 shear times) by solving the basic Eqs. (5)-(10) using the spectral SNOOPY code [49]. The mean magnetic field $\mathbf{B}_{\mathbf{0}}$ is conserved with time, because the domain-averaged fluctuating (turbulent) fields, as we checked, remain zero, $\langle\mathbf{u}\rangle=\langle\mathbf{b}\rangle=0$, during the whole run, thanks to the shearing box boundary conditions. SNOOPY is a general-purpose code, solving HD and MHD equations, including shear, rotation, weak compressibility, and several other physical effects. It is based on a spectral (Fourier) method allowing for the drift of harmonics in $\mathbf{k}$ space due to mean flow (i.e., the shearing box boundary conditions are implemented in the code). The Fourier transforms are computed using the FFTW 3 library. Nonlinear terms are computed using a pseudospectral algorithm [50], and antialiasing is enforced using the " $3 / 2$ rule." Time integration is performed by a third-order Runge-Kutta scheme for nonlinear terms, whereas an implicit scheme is used for viscous and resistive terms. This spectral scheme uses a periodic remap algorithm in order to continually follow the smallest wave number of the system in the sheared frame moving with the flow. The code has been tested and extensively used in a number of fluid dynamical and astrophysical contexts (see, e.g., Refs. [35,51-56]).

Figure 1 shows the time development of the domainaveraged perturbed kinetic, $\left\langle E_{K}\right\rangle$, and magnetic, $\left\langle E_{M}\right\rangle$, energies as well as the Reynolds $\left\langle u_{x} u_{y}\right\rangle$ and Maxwell $-\left\langle b_{x} b_{y}\right\rangle$ stresses. At the early stage of evolution, they all increase as a result of linear transient growth of separate SFHs contained in the initial conditions. Then, after about 250 shear times, on reaching sufficient amplitudes in the nonlinear regime,
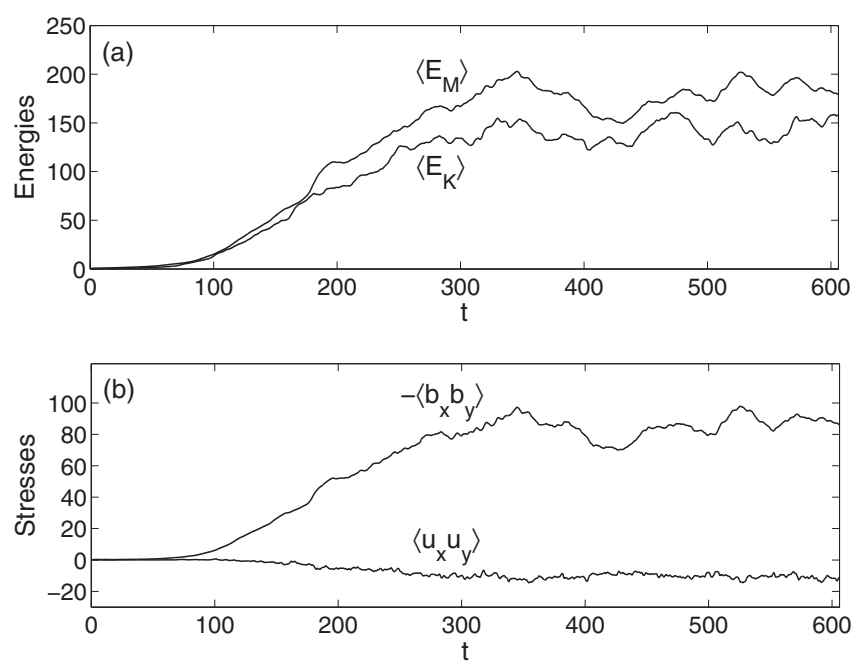

FIG. 1. Evolution of the domain-averaged (a) perturbed kinetic, $\left\langle E_{K}\right\rangle$, and magnetic, $\left\langle E_{M}\right\rangle$, energies as well as (b) the Reynolds and Maxwell stresses in the fiducial run. Data have been boxcar-averaged over 60 shear times to make the plot readable. In the beginning, all these quantities steadily grow as a result of shear-induced transient amplification of separate SFHs. Then, at about $t=250$, the amplification saturates to a quasisteady turbulent state that persists till the end of the run. The magnetic energy is a bit higher than the kinetic one and the positive Maxwell stress dominates over the negative Reynolds stress.

the energies and stresses settle down to a quasisteady state of sustained turbulence (see Fig. 2) that does not decay and persists until the end of the simulation at $t_{f}=600$. In this state, the kinetic and magnetic energies are comparable: the ratio of their domain- and time-averaged over the whole quasisteady state values (denoted here and below, for the stresses, by double angle brackets) is $\left\langle\left\langle E_{M}\right\rangle\right\rangle /\left\langle\left\langle E_{K}\right\rangle\right\rangle=1.28$; that is, there is a near-equipartition of the energy between kinetic and magnetic components. The Maxwell stress is much larger than the Reynolds stress, indicating that the turbulent transport and energy extraction from the mean flow are dominated by the magnetic field perturbations. The average of the domainaveraged Maxwell stress over the last 350 shear times is positive, $\left\langle\left\langle-b_{x} b_{y}\right\rangle\right\rangle=84.5$, while that of the domain-averaged Reynolds stress is negative, $\left\langle\left\langle u_{x} u_{y}\right\rangle\right\rangle=-10.4$. As shown by Eq. (13), the domain-averaged total stress must necessarily be positive for maintenance of turbulence and therefore it is the Maxwell stress that plays a decisive role in this process: counteracting dissipation, it ensures continuous feeding and sustenance of the turbulence at the expense of the mean shear flow.

The structure of the velocity and magnetic field in the quasisteady turbulent state (at $t=490$ ) is depicted in Fig. 2. These fields are chaotic, with $u_{y}$ and $b_{y}$ [Figs. 2(b) and 2(d)] having more elongated features in the $y$ direction due to shear compared to $u_{x}$ and $b_{x}$ [Figs. 2(a) and 2(c)]. At this time, the normalized fluctuating velocity and magnetic field are comparable, $\left\langle u_{x}^{2}\right\rangle=87.68,\left\langle u_{y}^{2}\right\rangle=178.73,\left\langle b_{x}^{2}\right\rangle=$ $113.17,\left\langle b_{y}^{2}\right\rangle=238.64$, and are much larger than their corresponding initial values. Also, the $y$ components are larger than the $x$ ones: $\left\langle u_{x}^{2}\right\rangle<\left\langle u_{y}^{2}\right\rangle,\left\langle b_{x}^{2}\right\rangle<\left\langle b_{y}^{2}\right\rangle$, which holds throughout 
(a)

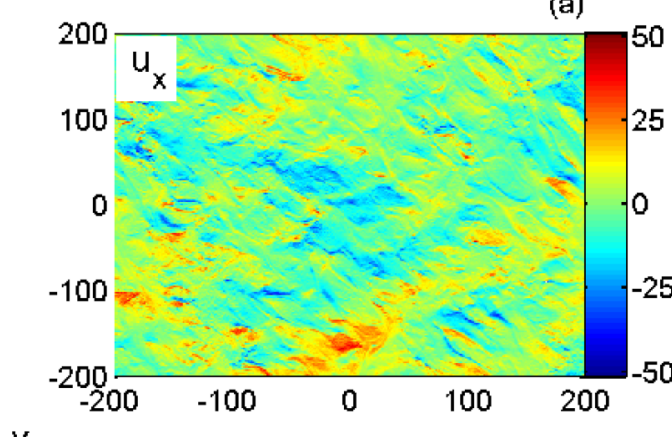

$y$

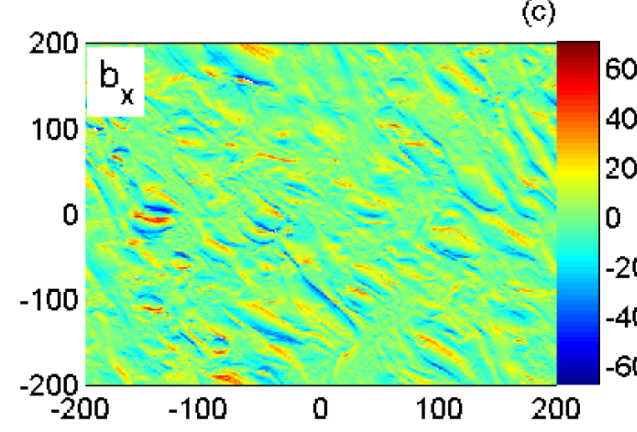

(b)

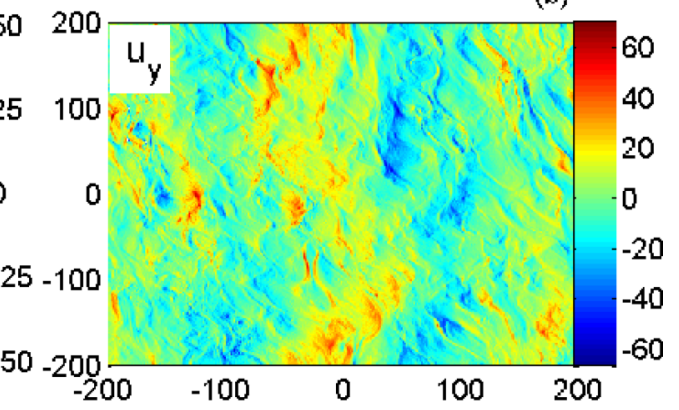

(d)

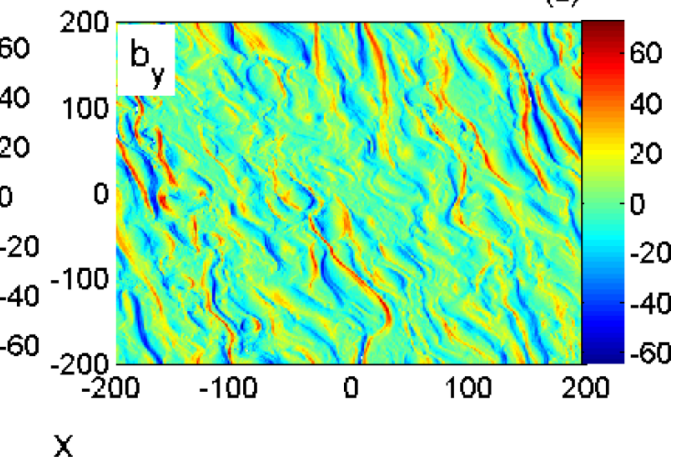

FIG. 2. (Color online) Distribution of (a), (b) the velocity and (c), (d) the magnetic field components in the ( $x, y$ ) plane in the fully developed quasisteady turbulence at $t=490$. This state is fairly nonlinear: $u_{x}$ and $u_{y}$ vary within limits comparable to the domain-averaged velocity of the background flow (in nondimensional units $\left\langle\left|U_{0}\right|\right\rangle=L_{x} / 4=100$ ), while $b_{x}$ and $b_{y}$ are much larger than the background magnetic field $B_{0 y}=1$. Structures in the $u_{y}$ and $b_{y}$ fields are elongated in the $y$ direction due to shear.

the run. Within the domain, $u_{x}$ and $u_{y}$ reach maximum values $\left|u_{x}\right|_{\max }=51.53$ and $\left|u_{y}\right|_{\max }=70.15$ comparable to the average background flow velocity, $\left\langle\left|U_{0}\right|\right\rangle=L_{x} / 4=100$, and the $b_{x}$ and $b_{y}$ have grown much larger, $\left|b_{x}\right|_{\max }=70.61$ and $\left|b_{y}\right|_{\max }=72.78$, than the mean field $B_{0 y}=1$. So, this quasisteady MHD turbulence can be viewed as being strongly nonlinear and weakly magnetized, since $\left\langle\mathbf{b}^{2}\right\rangle^{1 / 2} \gg B_{0 y}$.

The general behavior of the domain-averaged kinetic and magnetic energies and stresses with time obtained here in the $2 \mathrm{D}$ case is qualitatively consistent with that typically found in similar, but 3D, simulations of MHD turbulence driven by the magnetorotational instability (MRI) in local models of accretion disks with a net toroidal magnetic field along the disk flow $[31,33,34]$, as in the present setup. In both cases, there are no exponentially growing modes in the considered unbounded constant shear flows in the classical sense of linear stability analysis $[18,19]$; i.e., the flows are spectrally stable. In such flows, perturbations can grow only transiently during finite times [20,38,39], which is thought to be a key factor for the onset of subcritical turbulence [13-15]. One of the basic characteristics of subcritical transition is its sensitivity to the initial perturbation amplitude (e.g., Refs. [3,12,53,57]), which is also observed here. We found that there exists a critical amplitude for initial velocity and magnetic field perturbations (at a given $L_{x}, \mathrm{Re}$, and $\mathrm{Rm}$ ) below which turbulence is absent: there is only transient amplification insufficient to trigger transition, which eventually decays due to dissipation. By contrast, for initial amplitudes higher than the critical value a turbulent transition does occur after a phase of large enough transient growth, as is also evident in Fig. 1. Specifically, at $\operatorname{Re}=\mathrm{Rm}=5$, adopted here, the critical amplitude turned out to be $\left\langle\mathbf{u}^{2}\right\rangle_{\text {crit }}^{1 / 2}=\left\langle\mathbf{b}^{2}\right\rangle_{\text {crit }}^{1 / 2}=0.34$ (for the same type of initial noise spectrum for both velocity and magnetic field perturbations), and in the fiducial run we accordingly selected the initial rms amplitudes $(=0.84)$ larger than this in order to achieve turbulent regime. This confirms that the turbulence we study here is subcritical, however, we have not explored the transition process, that is, have not pinned down the critical transition amplitude for different values of the system parameters (domain size, Reynolds numbers, etc.) in more detail. The problem of subcritical transition in MHD shear flows deserves a special investigation in its own right, but in the present analysis we are mainly interested in the properties of the resulting self-sustaining turbulence itself once it has settled into a quasisteady state. The underlying physics of the onset and sustenance of subcritical turbulence in spectrally stable HD shear flows-the bypass concept-has been extensively studied in a number of papers (see, e.g., Refs. [13,15] for a review), but extension to MHD turbulence in spectrally stable magnetized shear flows, to the best of our knowledge, has not been systematically investigated yet. The equilibrium flow considered here with a linear spanwise shear of mean velocity and streamwise magnetic field is the simplest but important example of such spectrally stable magnetized shear flows that allows us to grasp specific processes determining the onset, self-sustenance, and spectral characteristics of MHD turbulence in this kind of flow. Deeper insight into the dynamics of such subcritical MHD turbulence can be gained by performing an analysis in spectral space. 


\section{TURBULENCE BEHAVIOR IN THE SPECTRAL PLANE}

In this section, we focus on the analysis of the dynamics of the quasisteady turbulent state in the Fourier plane. We now explicitly calculate the individual terms in Eqs. (21) and (22), which were classified and described in Sec. II, using the simulation data. The SNOOPY code, being of the spectral type, is particularly useful for this purpose, as it allows us to directly extract Fourier transforms from the data.

Before proceeding to spectral analysis, we note that generally a turbulent field and hence its Fourier transform are quite noisy. To remove this noise and extract valuable information on the trends in the turbulence dynamics, all Fourier transforms (spectra) presented below are averaged over 80 shear times. The interval between two successive dumps in the code was set to 1 shear time, so the averaging is represented by 80 snapshots. From now on we concentrate on the evolution after the quasisteady saturated nonlinear state has set in (i.e., at $t \gtrsim 250$ ), so we can choose the starting moment for averaging arbitrarily over the duration of this state, since the result is practically independent of this moment by virtue of the quasisteadiness of the process.

\section{A. Energy spectra}

Figure 3 shows the time-averaged spectra of the kinetic and magnetic energies in the $\mathbf{k}$ plane that have been established in the quasisteady turbulent state. Note that both spectra are strongly anisotropic, with the magnetic energy spectrum being broader than the kinetic energy one. For $k \gtrsim 0.5$, they have a similar elliptical shape inclined to the $k_{x}$ axis, whereas at $k \lesssim 0.5$ these spectra differ in structure: isolines for the magnetic energy divide into two sets of ellipses near the center with the same inclination. This indicates that SFHs with $k_{x} / k_{y}>0$ have more energy than those with $k_{x} / k_{y}<0$ at fixed $k_{y}$. Since $\beta \gg 1$, the effect of the mean flow shear prevails over that of the mean magnetic field, which leads us to suppose that the anisotropy of these spectra might be primarily due to shear [58]. These features of the kinetic and magnetic energy spectra, which clearly distinguish them from typical turbulent spectra in the classical shearless case [25], arise as a consequence of the specific way in which the terms of linear and nonlinear origin in Eqs. (21) and (22) operate in the $\mathbf{k}$ plane. We show below that these terms are anisotropic over wave numbers due to shear, resulting in a new phenomenon - the transverse cascade of power in the spectral plane-compared to the classical (isotropic) case.

The above time-averaged 2D spectra integrated over the angle in the $\mathbf{k}$ plane, $\bar{E}_{K, M}^{(k)}=k \int_{0}^{2 \pi} \bar{E}_{K, M} d \phi$, and represented as a function of $k$ are shown in Fig. 4. From intermediate wave numbers $k \sim 0.2$ up to dissipation wave numbers $k \sim k_{D}=$ 2.24, both one-dimensional (1D) spectra exhibit power-law dependence on $k$, however, with different spectral indices: the kinetic energy spectrum is well fitted by $k^{-1.4}$, and the magnetic energy spectrum by $k^{-2}$. At these wave numbers, the spectral density of the magnetic energy is higher than that of the kinetic one, but at smaller $k \lesssim 0.2$ it decreases and becomes less than the kinetic one, both deviating from the power law. These power-law parts of the spectra clearly differ from the typical Iroshnikov-Kraichnan (IK) spectrum,
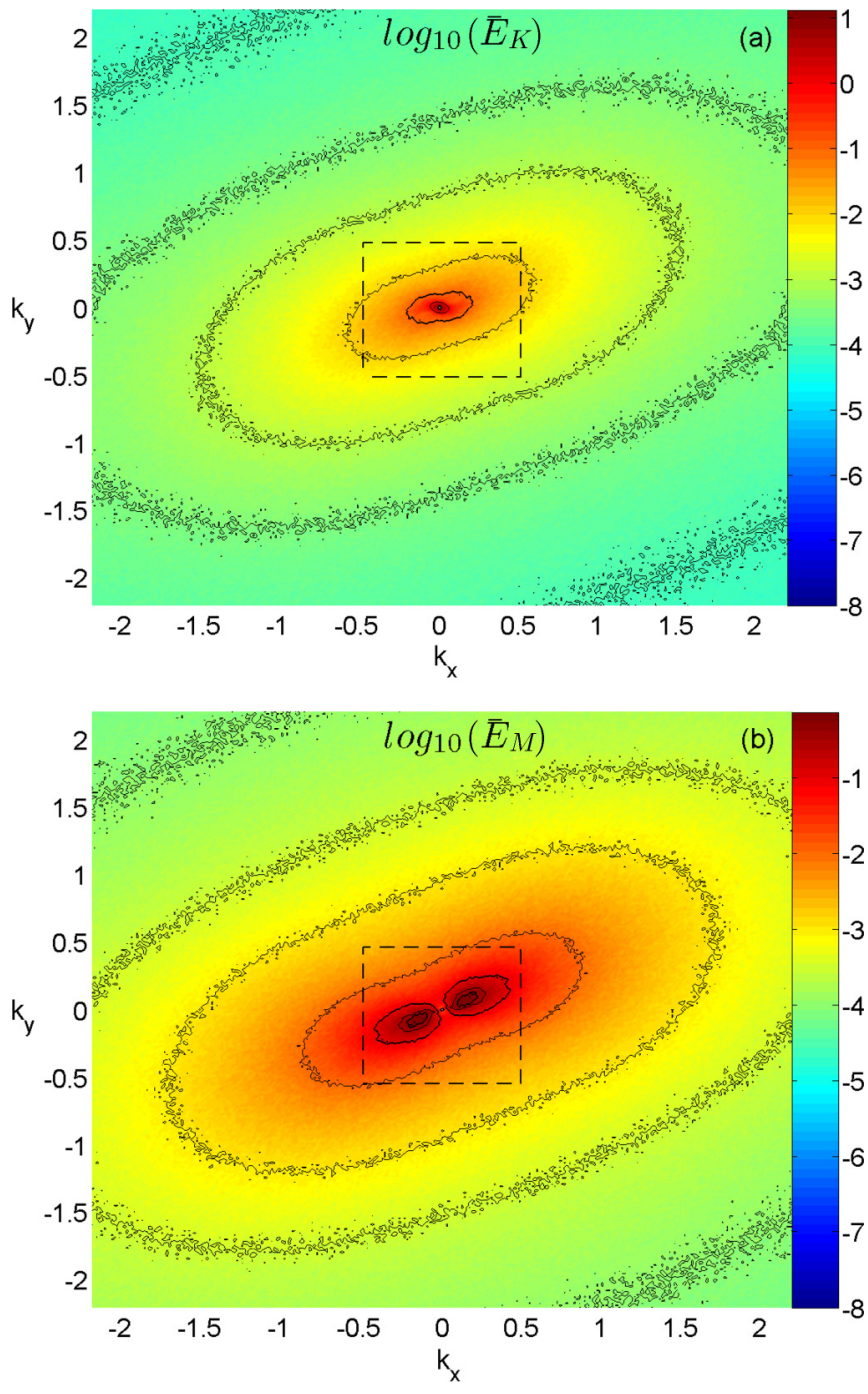

FIG. 3. (Color online) Time-averaged (a) kinetic and (b) magnetic energies' spectra in the $\mathbf{k}$ plane pertaining to the quasisteady turbulent state. These time averages are done over 80 shear times, as described in the text. Isolines correspond to (a) the values $-4,-3.5,-3,-2,-1$, and 0 of $\log _{10}\left(\bar{E}_{K}\right)$ and (b) to the values $-4,-3.5,-3,-2,-1,-0.5,-0.3$, and 0 of $\log _{10}\left(\bar{E}_{M}\right)$. Both spectra are anisotropic, having larger power at the $k_{x} / k_{y}>0$ side. The kinetic energy spectra is more concentrated at smaller wave numbers than the magnetic one. The dashed rectangle in each plot encloses the region of major activity of the dynamical terms in Eqs. (21) and (22), which are shown in Fig. 5.

$k^{-1.5}$, characteristic of classical 2D and 3D MHD turbulence without background shear flow [25], though the kinetic energy spectrum is still close to it. Different spectra of kinetic and magnetic energies, following approximately power-laws (though, with kinetic energy spectrum somewhat coincident with the IK one), are also present in analogous 3D simulations of MRI-driven MHD turbulence in the shearing box model of a disk $[35,45,59]$. However, it was pointed out in those studies that in the presence of differential rotation (shear) and weak magnetization $(\beta \gg 1)$ associated with disk flows, which are in fact also shared by the 2D MHD shear flow considered here, classical Kolmogorov or IK phenomenology 


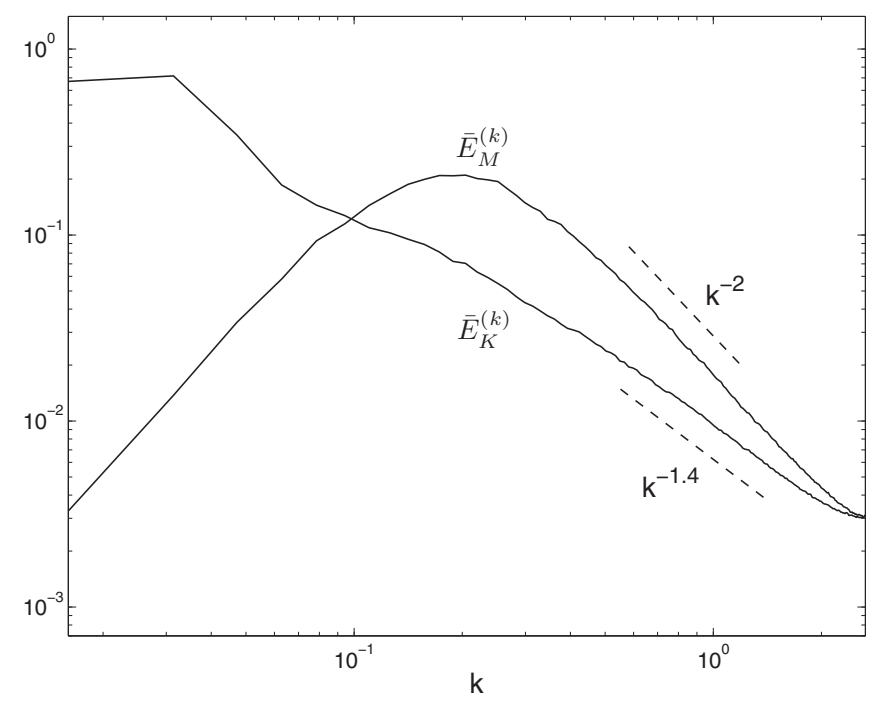

FIG. 4. Kinetic and magnetic energy spectra from Fig. 3 integrated over the angle in the $\mathbf{k}$ plane and plotted as a function of $k$. From intermediate, $k \sim 0.2$, to dissipation, $k \sim k_{D}=2.24$, wave numbers, a power-law behavior is observed in both spectra, though with different spectral indices: $k^{-1.4}$ for the kinetic and $k^{-2}$ for the magnetic energies.

is generally not applicable to turbulence dynamics, because due to shear, energy injection from the mean flow into turbulence can occur over a broad range of length scales available in the flow, from the largest scale down to the dissipation scale, which in turn prevents the development of the proper inertial range of a spectrum in the classical sense (see also Refs. [32,35]). So, the spectra obtained in those disk simulations, despite being of the power-law type, are in fact determined by the interplay between injection terms due to the linear MRI, operating over a range of wave numbers, and nonlinear terms in spectral space. The situation is similar in the present problem. As shown below, the action of the energy injection terms $I_{K}$ and, especially, $I_{M}$ extends over a range of wave numbers in the $\mathbf{k}$ plane and is remarkably anisotropic [see Figs. 5(a), 5(b), and 6]. As noted above, these terms are responsible for the linear transient amplification of SFHs and energy extraction from the mean flow, so in this respect they play a similar role, i.e., supply turbulence with energy, in our nonrotating case as the (transient) MRI in rotating disk flows. Moreover, we demonstrate that there exists a new phenomenon-the transverse nonlinear cascade of spectral energy density - resulting from this anisotropy and, ultimately, from shear. These new features are not common to shearless MHD turbulence and hence it is not surprising that Kolmogorov or IK theory cannot adequately describe shear flow turbulence.

We have presented the energy spectra from two perspectives: fully in the $\mathbf{k}$ plane in Fig. 3 and their angle-integrated (over shells of constant $|\mathbf{k}|$ ) versions in Fig. 4; the former is obviously more informative than the latter. We emphasize that angle integration of turbulent spectra and transfer functions when they are anisotropic in the wave-number plane might lead to the loss of essential information on the detailed nonlinear dynamics, so we take a more general strategy of
Ref. [16] and represent energy spectra as well as injection and nonlinear transfer terms in full in the $\mathbf{k}$ plane, in contrast to previous related studies of MHD turbulence in shear flows considering either such angle-integrated or reduced 1D spectra (e.g., Refs. [32,35,41,45]). This allows us to obtain a complete dynamical picture and understanding of the nature of subcritical MHD turbulence in the presence of mean flow shear.

\section{B. Spectra of energy injection: $I_{K}$ and $I_{M}$}

To better understand the character of the above anisotropic kinetic and magnetic energy spectra and nonlinear transfers, in Fig. 5 we present the distribution of the time-averaged kinetic and magnetic injection functions, $I_{K}$ and $I_{M}$, cross terms, $I_{K-M}$ and $I_{M-K}$, and nonlinear transfer terms, $N_{K}$ and $N_{M}$, in the $\mathbf{k}$ plane in the quasisteady turbulent state. From this figure it is seen that these terms differ in magnitude, and like the spectral energies, all exhibit anisotropy over wave numbers, that is, depend on the wave-vector angle. $I_{K}$ is mostly concentrated at small wave numbers, $k \lesssim 0.1$ [Fig. 5(a)], being positive at $k_{x} / k_{y}<0$ [white (red and yellow) regions], where it increases the kinetic energy of SFH, and negative at $k_{x} / k_{y}>0$ [darkgray (blue) regions], where it takes kinetic energy from $\mathrm{SFH}$ and gives it back to the flow. A net contribution of $I_{K}$ over all wave numbers is, however, negative (i.e., $\left\langle u_{x} u_{y}\right\rangle<0$ ). On the other hand, $I_{M}$ mostly operates at larger wave numbers, $0.05 \lesssim k \lesssim 0.5$ [Fig. 5(b)], and is dominant and positive on the $k_{x} / k_{y}>0$ side [white (red and yellow) regions], where it supplies SFH with magnetic energy. The net result of $I_{M}$ over all wave numbers is a positive energy gain for perturbations (i.e., $\left\langle-b_{x} b_{y}\right\rangle>0$ ), which prevails over the net negative effect of $I_{K}$, as is also evident in Fig. 1(b), and maintains turbulence. So, energy input for perturbation SFHs is provided by the magnetic source term $I_{M}$, which operates over a much broader region in the $\mathbf{k}$ plane than $I_{K}$ does. We checked that such a dependence of kinetic and magnetic energy injection terms on wave numbers, in fact, is also seen for the linear evolution of SFH; i.e., when the SFH drifts along the $k_{x}$ axis due to shear, its kinetic energy first increases at $k_{x} / k_{y}<0$, then decreases after crossing the point $k_{x}=0$, while its magnetic energy starts to increase at $k_{x} / k_{y}>0$ during a few shear times and then continues to oscillate at Alfvén frequency, $\omega_{A}=u_{A} k_{y}$, and constant amplitude (provided dissipation is neglected).

The linear cross terms, $I_{K-M}$ and $I_{M-K}$ [Figs. 5(c) and 5(d)], are small compared to both $I_{K}, I_{M}$ and nonlinear $N_{K}, N_{M}$ terms. In the spectral plane, the action of these terms is somewhat opposite to that of the corresponding injection terms. $I_{K-M}$ lowers the kinetic energy at small wave numbers but increases it at intermediate and large wave numbers on the $k_{x} / k_{y}>0$ side, while $I_{M-K}$ lowers the magnetic energy at intermediate and large wave numbers in the same quadrant and increases at small wave numbers. As noted above, these cross terms cancel out in the total energy, Eq. (24), and, because they are much smaller than the other dynamical terms, do not play any major role in the energy balance in Eqs. (21) and (22) too.

The difference between the injection wave numbers for the kinetic and magnetic energies is demonstrated more clearly in Fig. 6, showing these injection, nonlinear transfer, and dissipation terms angle-integrated in the k plane, $I_{K}^{(k)}, N_{K}^{(k)}, D_{K}^{(k)}$ 

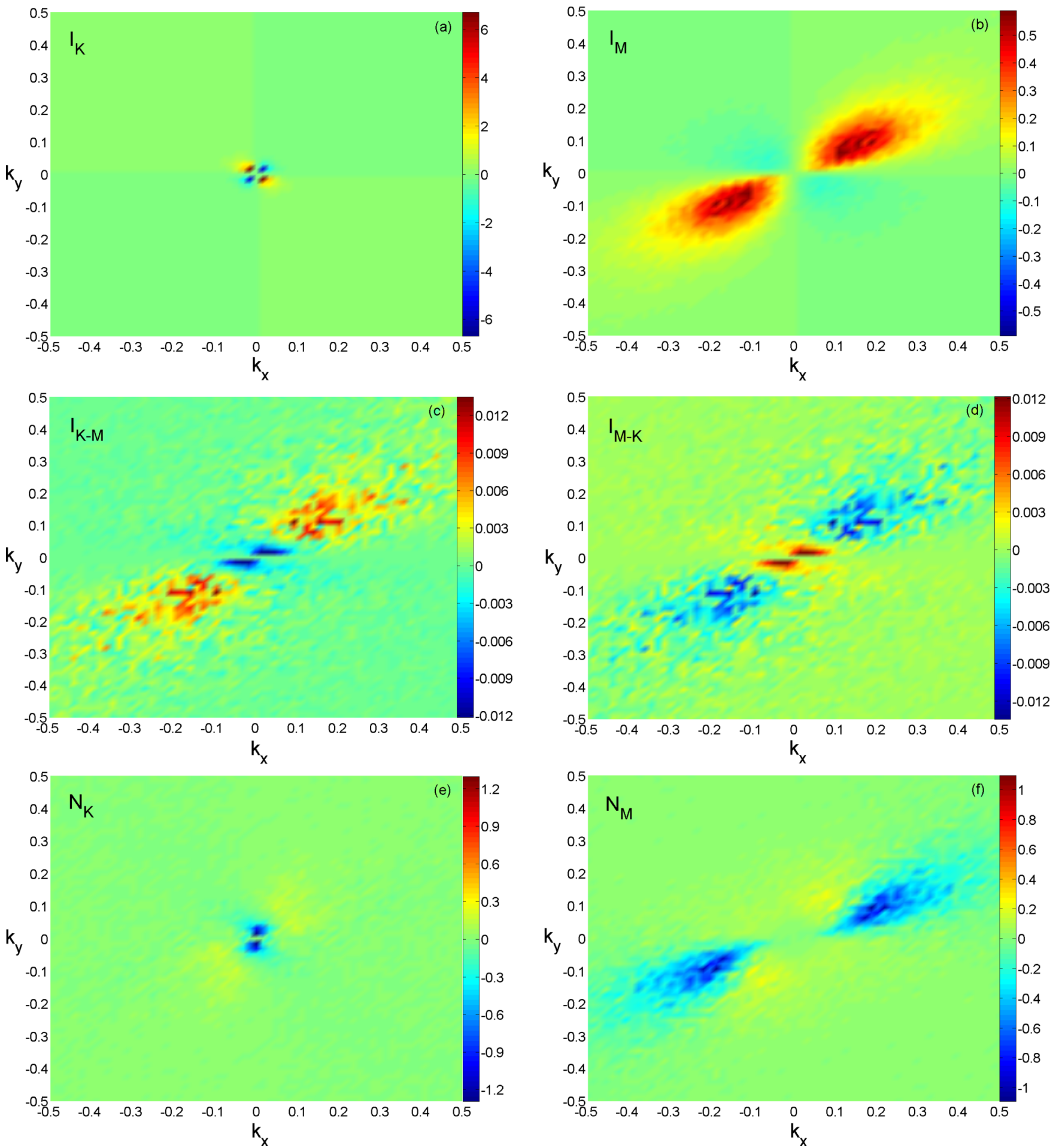

FIG. 5. (Color online) Maps of the time-averaged (a) kinetic, $I_{K}$, and (b) magnetic, $I_{M}$, energy injection terms, (c), (d) the cross terms $I_{K-M}, I_{M-K}$, and the (e) kinetic, $N_{K}$, and (f) magnetic, $N_{M}$, nonlinear transfer terms in the $\mathbf{k}$-plane in the state of quasisteady turbulence. The time averages are obtained over an interval of 80 shear times (from 472 to 552 shear times), as described in the text. Kinetic energy injection mostly occurs at small wave numbers, $k \lesssim 0.1$, and on the $k_{x} / k_{y}<0$ side where $I_{K}>0$, while magnetic energy injection occurs mostly at intermediate wave numbers, $0.05 \lesssim k \lesssim 0.5$, on the $k_{x} / k_{y}>0$ side where $I_{M}>0$, overall it is dominant over $I_{K}$; i.e., energy injection into turbulence appears to be due mainly to the Maxwell stresses. The $N_{K}$ and $N_{M}$ terms transfer, respectively, the spectral kinetic and magnetic energies anisotropically (transversely) in the wave-number plane, away from regions where they are negative, $N_{K}<0, N_{M}<0$ [dark gray and black (blue)], to regions where they are positive, $N_{K}>0, N_{M}>0$ [light gray and white (yellow)]. The nonlinear terms are comparable to the injection terms and both are about two orders of magnitude larger than the cross terms. 

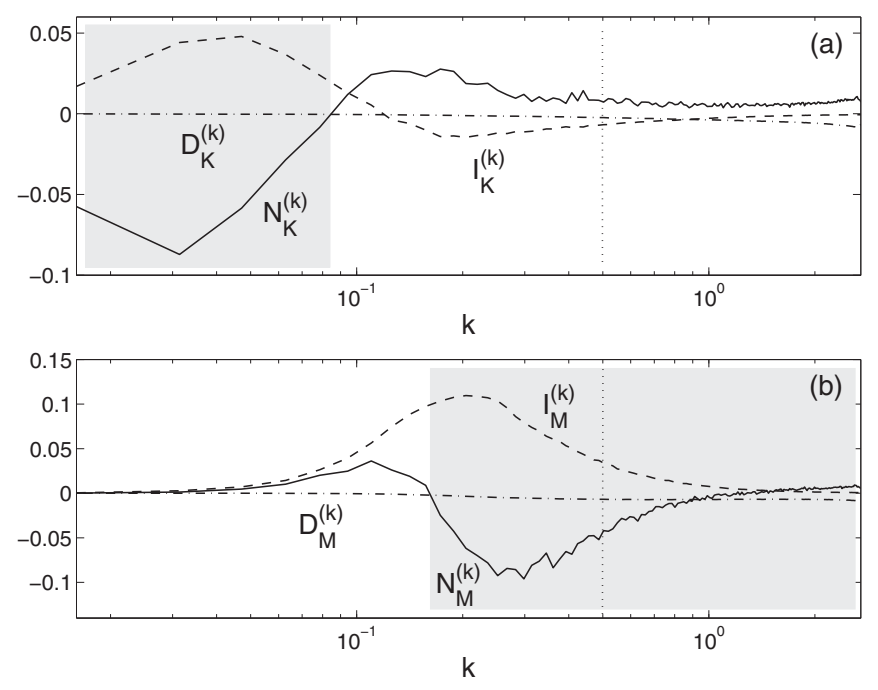

FIG. 6. Kinetic and magnetic injection and nonlinear transfer terms from Fig. 5 as well as dissipation terms integrated over the angle in the $\mathbf{k}$ plane-(a) $I_{K}^{(k)}, N_{K}^{(k)}, D_{K}^{(k)}$ and (b) $I_{M}^{(k)}, N_{M}^{(k)}, D_{M}^{(k)}$-and represented as a function of $k$. Injection terms (dashed lines) operate over a range of wave numbers, overlapping with nonlinear terms (solid lines). The magnetic energy injection is larger than the kinetic one. Both viscous and resistive dissipation (dot-dashed lines) are relatively important only at $k>k_{D}=2.24$. The reference dotted vertical line marks the maximum wave number, $k=0.5$, of the domains in Fig. 5. Shaded (gray) regions correspond to wave numbers at which $N_{K}^{(k)}<0, N_{M}^{(k)}<0$, and hence the kinetic and magnetic energies, respectively, are transferred, on average, away from these wave numbers due to nonlinearity.

[Fig. 6(a)] and $I_{M}^{(k)}, N_{M}^{(k)}, D_{M}^{(k)}$ [Fig. 6(b)], and represented as a function of $k$. It is seen in this figure that the range of wave numbers, where the injection terms are at work, extends from the smallest wave numbers in the domain, $k_{x \text {, min }}$, up to $k \sim 1$ comparable to the dissipation wave number $k_{D} . I_{K}^{(k)}$ is positive at small wave numbers, reaching a maximum at $k \approx 0.05$, then becomes negative and vanishing at $k>0.12$ (i.e., no longer injects kinetic energy). On the other hand, $I_{M}^{(k)}$ is positive and hence creates the turbulence's magnetic energy at all wave numbers, reaching a maximum at $k \approx 0.2$, which is about twice as large as that of $I_{K}^{(k)}$. Note in Fig. 6 that these injection and nonlinear transfer terms $N_{K}^{(k)}$ and $N_{M}^{(k)}$ widely overlap. This implies that in the presence of shear, there is not a single injection scale in the flow, as is usually assumed in classical turbulence theory, but instead, energy injection occurs all the way from the largest length scales down to the dissipation scale. Therefore, although power-law spectra for both the kinetic and the magnetic energies are found at $0.2 \lesssim k \lesssim 2$ (Fig. 4), they still cannot be considered as being a proper inertial range, since energy is injected at these intermediate scales (see also Refs. [32,35] for a similar situation in the MRI-driven turbulence, where the injection of energy, drawn from the mean flow, into turbulence occurs over a range of scales at which nonlinear transfers operate as well). In Fig. 6, it is also seen that in this wave-number range, the dissipation terms are much smaller than the injection and nonlinear transfer terms, so this part of the energy spectra are in fact formed mainly as a result of the combined action of the linear injection and nonlinear cascade.

\section{Nonlinear transfers $N_{K}$ and $N_{M}$ : The essence of the transverse cascade}

We now move to describing the nonlinear kinetic and magnetic transfer functions. As noted above, they do not represent a new source of total energy for turbulence, but only act to redistribute kinetic and magnetic spectral energies, which are extracted from the mean flow, over wave numbers and, in cooperation with injection terms, determine the characteristics of spectra. So, our primary goal is to understand how the nonlinear transfer terms work and, consequently, in which directions energies cascade in the Fourier plane in the presence of background shear. As mentioned in Sec. I, for a purely HD constant shear (Couette) flow, which is spectrally stable, it was shown in Ref. [16] that nonlinear transfer function is anisotropic in the $\mathbf{k}$ plane, i.e., depends on the polar angle due to shear and, as a consequence, leads to redistribution of the spectral energy over wave-vector angles. This relatively new process, termed the angular, or transverse cascade of energy, has been shown to be essential for the maintenance of the subcritical nonlinear state in this flow via the bypass mechanism. Actually, identification of the transverse cascade of energy has been made possible by virtue of representation of the dynamics fully in the 2D spectral plane, without performing angle integration, which would result in washing out a key element of this process: the angular dependence (anisotropy) of the transfer functions' spectra. The findings in that paper indicate that in HD shear flows, along with the direct and inverse cascades quite well established in turbulence theory, a new, transverse type of cascade can also take place, which, in fact, appears to be as important as the former. Based on these results, in the present paper we generalize a spectral analysis of nonlinear dynamics given in Ref. [16] for the HD constant shear flow to the MHD constant shear flow considered here, with the aim of understanding the mechanism responsible for the sustenance of the subcritical MHD turbulence in question. Specifically, we examine whether there exists a cooperative action of any kind between energy-injecting linear and nonlinear transfer terms, like that occurring in HD shear flows, capable of sustaining perturbations in spectrally stable MHD shear flows.

Figures 5(e) and 5(f) show the distribution of the timeaveraged kinetic, $N_{K}$, and magnetic, $N_{M}$, nonlinear transfer functions with wave numbers in the quasisteady turbulence, alongside the injection terms, in order to easily see their cooperative (correlated) action with the latter. As mentioned above, both $N_{K}$ and $N_{M}$ are strongly anisotropic, i.e., depend on the polar angle in the $\mathbf{k}$ plane. This anisotropy has qualitatively the same character as that of $I_{K}, I_{M}, I_{K-M}$, and the $2 \mathrm{D}$ energy spectra in Fig. 3; that is, the spectra of all these are inclined towards the $k_{x}$ axis due to shear. To bring out this angular dependence more clearly, we integrated $I_{K}, I_{M}$ and $N_{K}, N_{M}$ over $k$, from the smallest $k_{\min }=k_{x, \min }$ to the largest $k_{\max }=k_{x, \max }$ values in the domain,

$$
I_{K, M}^{(\theta)}=\int_{k_{\min }}^{k_{\max }} I_{K, M} k d k, \quad N_{K, M}^{(\theta)}=\int_{k_{\min }}^{k_{\max }} N_{K, M} k d k
$$



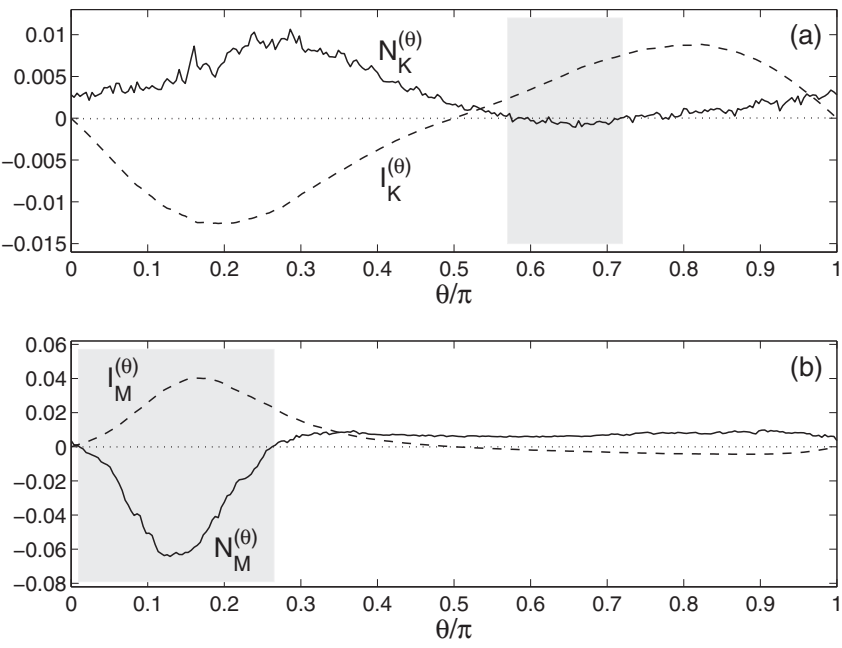

FIG. 7. Kinetic and magnetic injection and nonlinear transfer terms from Fig. 5 integrated over $k$ - (a) $I_{K}^{(\theta)}, N_{K}^{(\theta)}$ and (b) $I_{M}^{(\theta)}, N_{M}^{(\theta)}-$ and represented as a function of the wave-vector polar angle $\theta$ (angles $\pi<\theta<2 \pi$ correspond to complex conjugates and are not shown here). These plots clearly demonstrate the angular dependence (anisotropy) of both the injection (dashed lines) and the nonlinear transfer (solid lines) terms. Shaded (gray) regions correspond to angles at which $N_{K}^{(\theta)}<0, N_{M}^{(\theta)}<0$, and hence kinetic and magnetic energies, respectively, are transferred from these angles to other angles due to nonlinearity; that is, a new phenomenon-a transverse (angular) cascade of energy - takes place.

and represent them as functions of the polar angle $\theta$ in Fig. 7. While the above-defined $N_{K}^{(k)}$ and $N_{M}^{(k)}$ describe energy transfers in the direction of $\mathbf{k}, N_{K}^{(\theta)}$ and $N_{M}^{(\theta)}$ describe energy transfer along the azimuthal direction, perpendicular to $\mathbf{k}$.

As shown in Figs. 5-7, the distributions of $N_{K}$ and $N_{M}$ over wave numbers differ, leading to different types of cascades for the kinetic and magnetic spectral energies. Since these quantities are symmetric with respect to a change $\mathbf{k} \rightarrow-\mathbf{k}$, without loss of generality, everywhere below we concentrate on the upper part $\left(k_{y}>0\right)$ of the $\mathbf{k}$ plane. $N_{K}$ mainly operates in two regions of the $\mathbf{k}$ plane: at small wave numbers, $k \lesssim 0.1$, where it is negative [dark-gray and black (blue) region with $N_{K}<0$ in Fig. 5(e), corresponding to the gray-shaded area with $N_{K}^{(k)}<0$ in Fig. 6(a)], and at intermediate wave numbers, $0.1 \lesssim k \lesssim 0.5$, on the $k_{x} / k_{y}>0$ side $(0 \leqslant \theta \leqslant \pi / 2)$, where it is positive [light-gray and white (yellow) region with $N_{K}>0$ ]; at all other wave numbers the kinetic transfer function is nearly zero. On the other hand, $N_{M}$ mainly operates at $0.05 \lesssim k \lesssim 1$ [see also Fig. 6(b)], is positive at $0.3 \pi \lesssim \theta \leqslant \pi$ [light-gray and white (yellow) region with $N_{M}>0$ in Fig. 5(f)], and is negative at $0 \leqslant \theta \lesssim 0.3 \pi$ [dark-gray and black (blue) region with $N_{M}<0$ ]; at all other wave numbers the magnetic transfer term is nearly zero. Note also that the distributions of $N_{K}$ and $N_{M}$ look somewhat similar to those of the linear exchange terms $I_{K-M}$ and $I_{M-K}$, respectively, but as noted above, the latter are two orders of magnitude smaller than the former.

By definition, these nonlinear transfer functions redistribute the corresponding spectral energies away from the regions in the $\mathbf{k}$ plane where they are negative to the regions where they are positive. The kinetic energy injection due to $I_{K}$ occurs, as described above, at small wave numbers $(k \lesssim 0.1)$ with $\pi / 2<\theta<\pi$, where $I_{K}>0$ [see also Figs. 6(a) and 7(a)], but the $N_{K}$ term is negative there, transferring kinetic energy away from these injection wave numbers to intermediate wave numbers, $k \gtrsim 0.1$, with $0 \leqslant \theta \leqslant \pi / 2$, where $N_{K}>0$. This picture of spectral kinetic energy transfer, or cascade towards larger wave numbers, is also evident in Fig. 6(a), where the angle-integrated $N_{K}^{(k)}$ changes from negative to positive at about $k=0.1$, consistent with the flow of kinetic energy away from $k \lesssim 0.1$ to $k \gtrsim 0.1$. The cascade behavior for the turbulent magnetic energy is different from that of the kinetic energy. The magnetic energy injection due to $I_{M}$ occurs at intermediate wave numbers $(0.05 \lesssim k \lesssim 1)$ for $0<\theta<\pi / 2$, where $I_{M}>0$ [see also Figs. 6(b) and 7(b)], but the $N_{M}$ term, which is mostly negative there, transfers the magnetic energy away from this injection region to its neighboring region on the left, with slightly smaller wave numbers but larger polar angles $0.3 \pi \lesssim \theta \leqslant \pi$, where $N_{M}$ is positive. This cascade of magnetic energy to smaller wave numbers is more clearly shown in Fig. 6(b), where the angle-integrated $N_{M}^{(k)}$ changes from positive to negative at around $k=0.17$, indicating the flow of magnetic spectral energy from $k \gtrsim 0.1$ to $k \lesssim 0.1$.

Thus, in shear MHD turbulence, the kinetic and magnetic energies are transferred both along the wave vector, corresponding to familiar direct and inverse cascades, and transversely (perpendicular) to it (i.e., over angles $\theta$ ). Just this second type of nonlinear cascade, better characterized by $N_{K}^{(\theta)}$ and $N_{M}^{(\theta)}$ (Fig. 7), is a new effect of shear and is discussed more in the next subsection; it is absent in classical shearless MHD turbulence.

As stressed in Ref. [16], the transverse cascade of energy appears to be a generic feature of nonlinear dynamics of perturbations in spectrally stable shear flows, so the conventional description of shear flow turbulence solely in terms of direct and inverse cascades, which leaves such a nonlinear transverse cascade out of consideration, might be incomplete and misleading. We emphasize that in the present case revealing the complete picture of these nonlinear cascade processes has become largely possible due to carrying out the analysis in the spectral plane. Because of the shear-induced anisotropy of cascade directions, only angle-integrated transfer functions in Fig. 6 (which are, in fact, typically used in most numerical studies of shear MHD turbulence; e.g., Refs. [32,35,41,45]), clearly, are not fully representative of the actual, more general nonlinear redistribution of the spectral energies in the $\mathbf{k}$ plane, which also includes transfer with respect to wave-vector angles: the transverse cascade.

\section{Interplay of the linear injection and nonlinear transverse cascade}

We have seen above that the nonlinear redistributions of spectral kinetic and magnetic energies over the wave-vector polar angle, $\theta$, in the $\mathbf{k}$ plane, termed the transverse cascade, are due to shear-induced dependence of the nonlinear transfer functions $N_{M}$ and $N_{M}$ on this angle. This can be better appreciated from Fig. 7, showing the $N_{K}^{(\theta)}$ and $N_{M}^{(\theta)}$ introduced in previous subsection. They exhibit different dependences over $\theta$, resulting in different characters of the transverse 
cascade for the kinetic and magnetic energies. Note the opposite relative trends between $I_{K}^{(\theta)}$ and $N_{K}^{(\theta)}$ [Fig. 7(a)] and between $I_{M}^{(\theta)}$ and $N_{M}^{(\theta)}$ [Fig. 7(b)] with respect to $\theta$ :

$$
\begin{aligned}
& I_{K}^{(\theta)} \leqslant 0 \quad \text { and } \quad N_{K}^{(\theta)}>0 \quad \text { at } 0 \leqslant \theta \leqslant \pi / 2, \\
& I_{K}^{(\theta)} \geqslant 0 \quad \text { and } \quad N_{K}^{(\theta)} \approx 0 \quad \text { at } \pi / 2<\theta \leqslant \pi .
\end{aligned}
$$

On the other hand,

$$
\begin{aligned}
& I_{M}^{(\theta)} \geqslant 0 \quad \text { and } \quad N_{M}^{(\theta)} \leqslant 0 \text { at } 0 \leqslant \theta \lesssim 0.3 \pi, \\
& I_{M}^{(\theta)} \approx 0 \quad \text { and } \quad N_{M}^{(\theta)}>0 \text { at } 0.3 \pi \lesssim \theta \leqslant \pi .
\end{aligned}
$$

This implies that the region of the $\mathbf{k}$ plane where SFHs are replenished with kinetic energy by nonlinearity (i.e., where $N_{K}>0$ ) lies on the right side of the kinetic energy injection region with $I_{K}>0$, whereas the region where SFHs are replenished with magnetic energy by nonlinearity (i.e., where $N_{M}>0$ ) lies on the left side of the magnetic energy injection region with $I_{M}>0$, as also shown in Fig. 5. As explained below, this specific arrangement of the injection and nonlinear redistribution areas for the magnetic energy in the spectral plane appears to be crucial to the sustenance of the turbulence.

After characterizing the specific activity of the linear injection and nonlinear transfer terms in the $\mathbf{k}$ plane associated with the presence of shear, we now consider the evolution of SFHs in quasisteady turbulence and identify a mechanism sustaining this state. As noted above, apart from these terms, Eqs. (21) and (22) also contain terms describing drift of SFHs in the spectral plane due to shear flow. In the upper half-plane $\left(k_{y}>0\right)$ we focus on, all SFHs drift along the $k_{x}$-axis direction and cross the injection and transfer regions in succession. Since the turbulence is quasisteady, these three basic processes involved in the spectral Eqs. (21) and (22)-linear drift of SFHs, energy injection, and nonlinear transfer-together with viscous and resistive dissipation, are in subtle balance, or cooperation, resulting in the closed (positive) feedback loop that energetically maintains this state. We interpret the workings of this loop as follows. Let us start the loop cycle. The nonlinear transfer functions $N_{K}$ and $N_{M}$ supply (from a previous cycle) SFHs with kinetic energy mainly at wave numbers with polar angles $0 \leqslant \theta \lesssim 0.6 \pi$ and $0.7 \pi \lesssim \theta \leqslant \pi$, where $N_{K}, N_{K}^{(\theta)}>0$, and magnetic energy at $0.3 \pi \lesssim \theta \leqslant \pi$, where $N_{M}, N_{M}^{(\theta)}>0$ [see Figs. 5(e), 5(f), and 7]. Then these SFHs drift along the $k_{x}$ direction and enter the injection regions, where $I_{K}>0$ and $I_{M}>0$. As a result, the kinetic energy of SFHs with $k_{y} \lesssim 0.1$ and the magnetic energy of SFHs with $k_{y} \gtrsim 0.05$ grow at the expense of the mean flow: just at this stage the kinetic and magnetic energies are being injected into the turbulence due to $I_{K}$ and $I_{M}$ from the mean flow. Then the SFHs move into the regions where $N_{K}<0$ and $N_{M}<0$, and hence these nonlinear terms now act to transfer part of the kinetic and magnetic energies from the amplified SFHs back, respectively, to the regions where $N_{K}>0$ and $N_{M}>0$, from which these SFHs started off, in this way regenerating new SFHs there (positive nonlinear feedback). Towards the end of the cycle, part of the original SFH's kinetic energy is returned to the mean flow, since $I_{K} \leqslant 0$ at $0 \leqslant \theta \leqslant$ $\pi / 2$, so effectively there is no net gain of the turbulent kinetic energy from the mean shear flow; the second part, which goes into the new SFHs, is taken from the magnetic energy via the nonlinear exchange by positive $N_{K}$ (at $k_{y} \gtrsim 0.1$ ); and the third part is gradually dissipated due to viscosity as the SFH drifts further towards larger wave numbers $\left(k \gtrsim k_{D}\right)$. So, during each cycle, the SFHs gain primarily magnetic energy from the mean flow due to the injection term $I_{M}$. Part of this magnetic energy is transformed by nonlinearity into the kinetic, as mentioned above, and the other part into the magnetic energies of the newly created SFHs. The rest of the magnetic energy is dissipated due to resistivity. As shown in Figs. 5(f) and 7(b), in the $\mathbf{k}$ plane, the magnetic injection region lies on the right side of the region of its nonlinear regeneration where $N_{M}>0$. As a consequence, these new (regenerated) SFHs will drift through the same cycle and the whole process of (magnetic) energy extraction from the mean flow will be repeated. In this way, a positive feedback loop - a cooperative interplay of the linear transient amplification and nonlinear transverse redistribution of the magnetic spectral energy-is established, ensuring the sustenance of a quasisteady turbulent state at the expense of the background flow energy. Such a constructive regeneration of those SFHs due to nonlinearity, which can extract shear flow energy during the linear transient amplification process, is the basis for the sustenance of subcritical turbulence in spectrally stable shear flows in the framework of the bypass concept [13].

We have seen that a principal role in the above-described MHD self-sustaining mechanism is played by magnetic field perturbations that actually feed turbulence: SFHs, which are able to extract energy from the shear flow by means of the Maxwell stresses (i.e., by $I_{M}$ ), are continuously repopulated by the nonlinear magnetic transfer term. This nonlinear positive feedback for the magnetic perturbations is probably related to the fact that the Maxwell stress has the "right" positive sign to supply turbulence [Fig. 1(b)]. By contrast, the injection region for the kinetic energy in the $\mathbf{k}$ plane lies to the left and below the main region of its nonlinear regeneration [at $0 \leqslant \theta \leqslant \pi / 2$, where $N_{K}>0$; see Figs. 5(e) and 7(a)]. As a result, the majority of new SFHs, drifting along the $k_{x}$ axis, cannot cross the injection region and thus continuously gain the kinetic energy from the flow; even the small fraction of new SFHs that can cross this region eventually returns the kinetic energy to the flow where $I_{K}<0$. In other words, the nonlinear feedback for the kinetic energy does not operate in a similar, constructive, manner as that for the magnetic energy. This may be related to the Reynolds stress being negative [Fig. 1(b)] and hence ineffective in feeding turbulence with kinetic energy. So, in the 2D MHD shear turbulence considered here, unlike the Maxwell stress, the Reynolds stress cannot provide the right sign for transport.

\section{DISCUSSION AND SUMMARY}

In this paper, we have studied the characteristics and self-sustaining mechanism of subcritical MHD turbulence in incompressible magnetized spectrally stable shear flows via DNS using the spectral code SNOOPY. We have examined how the background shear flow interacts with the turbulent fluctuations of the incompressible 2D MHD equations to produce a self-sustained turbulence. The analysis of the turbulence dynamics was carried out in the Fourier plane. To keep the problem as manageable as possible and, at the same time, not to omit key effects of shear on the dynamics of turbulence, 
as the base flow we took the simplest but important case of plane MHD Couette flow with linear shear and an imposed background uniform, weak, magnetic field parallel to it. This flow configuration is linearly stable (with decaying linear perturbations at long times) according to classical (modal) stability theory, and hence the only cause of transition to turbulence can be a linear transient amplification of (magnetic field) perturbations due to the non-normality associated with shear at streamwise wave numbers $k_{y}<S / u_{A}$. Consequently, the considered 2D MHD turbulence is subcritical by nature. To understand its sustaining mechanism, we Fourier transformed basic MHD equations and derived evolution equations for the perturbed kinetic and magnetic spectral energies in the wave-number plane. In these spectral equations, using the simulation results, we calculated individual terms, which are divided into two types: terms of linear and nonlinear origin. The terms of linear origin-the Maxwell and Reynolds stresses-are responsible for energy exchange between the turbulence and the mean flow through transient amplification of perturbation harmonics due to shear. However, as we have shown, only the positive Maxwell stress appears to be a dominant (magnetic) energy injector for the turbulence; it is much larger than the Reynolds stress, which has a negative sign and therefore does not contribute to the turbulent kinetic energy gain. Another linear term due to shear in these equations makes the spectral energies drift in the spectral plane parallel to the $k_{x}$ axis. The nonlinear terms, which do not directly draw the mean flow energy, act to transversely redistribute this energy in the Fourier plane, continually repopulating perturbation harmonics that can undergo transient growth. Thus, we have demonstrated that in spectrally stable shear flows, the subcritical MHD turbulent state is sustained by the interplay of linear and nonlinear processes: the first supplies energy for turbulence via the shear-induced transient growth mechanism of magnetic field perturbations (characterized by the Maxwell stresses), and the second plays the important role of providing a positive feedback that makes this transient growth process recur over long times and compensate for high- $k$ dissipation due to viscosity and resistivity.

This picture is consistent with the well-known bypass scenario of subcritical turbulence in spectrally stable shear flows [13] and differs fundamentally from the usual (supercritical) turbulence scenario, which is based on exponentially growing perturbations in a system that permanently supply turbulent energy and do not require nonlinear (positive) feedback for its sustenance. Such a cooperative action of linear transient growth and nonlinear transfer mechanisms relies on anisotropy of the energy spectra, injection, and nonlinear cascades in the spectral plane (see Fig. 5), which is ultimately attributable to the flow shear. This shear-induced anisotropy, i.e., the dependence of spectra and nonlinear transfers on the polar angle in the $\mathbf{k}$ plane, as we found and analyzed here in the case of MHD flows, appears to be inherent in shear flow turbulence; a similar anisotropy exists in HD shear flows (see Ref. [16] for details). It differs from the typical anisotropy of classical (shearless) MHD turbulence in the presence of a (strong) background magnetic field (e.g., Ref. [23]) and changes the classical view on nonlinear cascade processes: traditionally, the net action of nonlinear turbulent processes is interpreted as either a direct or an inverse cascade (e.g., Ref. [25]). Our analysis demonstrates, however, that in MHD shear flows, like HD ones, the dominant nonlinear process, resulting from the spectral anisotropy, is in fact the redistribution of perturbation SFHs over wave-vector angles. (Probably for this reason, in our simulations with background shear we did not observe the typical 2D coherent magnetic structures that grow via merging due to inverse cascade of magnetic helicity [60,61]). These anisotropic energy transfers in Fourier space have been termed nonlinear transverse redistribution, or the transverse cascade. In the considered flow, the nonlinear transverse cascade plays a vital role in the long-term sustenance of turbulence: it redistributes mainly magnetic spectral energy over different angles in the $\mathbf{k}$ plane such that to continually regenerate those harmonics which, drifting in the spectral plane, have the potential to undergo transient growth, extracting energy from the mean flow. This indicates that the transverse cascade of spectral (magnetic) energy appears to be characteristic of MHD turbulence in shear flows, so the conventional characterization of nonlinear MHD cascade processes in the presence of the flow shear in terms of direct and inverse cascades, which ignores the transverse cascade, should be generally incomplete and misleading. Identification of this new - transverse - type of nonlinear cascades and its role in the maintenance of shear MHD turbulence represents one of our main results.

We showed that as a result of anisotropy of nonlinear transfers in the $\mathbf{k}$ plane, kinetic and magnetic energy spectra are also highly anisotropic (see Fig. 3). These spectra integrated over the wave-vector angle exhibit power-law behavior for intermediate wave numbers, though with different spectral indices: $k^{-1.4}$ for the kinetic and $k^{-2}$ for the magnetic energies. Despite this, the angle-averaged spectra we found should not be regarded as truly inertial ranges, because the stresses inject kinetic and magnetic energies into turbulence over a broad range of wave numbers-from the largest scales in the domain down to the shortest scales comparable to the dissipation scale - well overlapping with the nonlinear transfer terms (see Figs. 6 and 7). So, these spectra are determined by the combined effect of linear injection and nonlinear transfer terms. This is in contrast to the usual forced turbulence case, where energy is injected (by external forcing) in a narrow wave-number band and subsequent development of spectra is due to nonlinearity only (e.g., Refs. [25,29,30]). As noted above, the energy injection by the stresses occurs through the transient amplification of perturbation Fourier harmonics due to shear, implying that the shear plays an important dynamical role at large and intermediate scales $\left(\gtrsim u_{A} / S\right)$. However, the angle averaging of anisotropic spectra (and also of transfer functions) in shear flows, as often done in similar cases, might lead to the loss of essential information about the spectral characteristics of shear turbulence because of its angular dependence too.

In the context of the spectral indices, it is interesting to point out that in some regions of the Earth's magnetotail, a magnetic energy spectrum with a slope close to that obtained here, $k^{-2}$, is observed [37]. It is hard to attribute this observational result to either the Kolmogorov or the IK spectra. This may suggest the influence of shear flow on the dynamics of the magnetotail turbulence and formation of its spectrum. The way we see it, definite conclusions can be drawn by performing a numerical 
analysis similar to that presented here for a specific 3D model configuration of the magnetotail.

In this paper, we have considered 2D dynamics, and a brief discussion of 3D MHD turbulence in magnetized shear flows is in order. According to the classical view, there is a fundamental difference in the nonlinear dynamics of $2 \mathrm{D}$ versus $3 \mathrm{D} \mathrm{HD}$ processes: 3D ones are characterized by a direct cascade of energy; 2D ones, by inverse cascade. By contrast, in MHD, the nonlinear dynamics of $2 \mathrm{D}$ and 3D processes are similar in the sense that cascade directions of characteristic quantities (energy, helicity, etc.) are identical (see, e.g., Ref. [25]). As for the transverse cascade analyzed in this paper, it occurs in HD as well as in MHD shear flows. It is well known that in HD shear flows, 2D turbulence is not maintained and dies out (without external forcing); i.e., inverse cascade modified by transverse cascade is unable to sustain turbulence (HD turbulence in shear flows is usually 3D). The present study demonstrates that, unlike HD shear flows, self-sustained 2D turbulence can exist in MHD shear flows owing to the transverse cascade. Being dependent on the shear, the transverse cascade is expected to occur and play an important role in the dynamics of 3D MHD shear turbulence too. But further studies should clarify whether the nonlinear dynamics with the third $z$ direction (perpendicular to the flow plane) represents a mere extension of the basic self-sustaining process described here in two dimensions or introduces a qualitatively new contribution. In any case, the transverse cascade will remain a vital ingredient in the self-sustenance of turbulence in three dimensions too. Although our analysis is limited to two dimensions, since these are the streamwise and shearwise directions, it allows us to bring out a basic mechanism underlying the self-sustenance (via interplay of linear transient amplification and nonlinear transverse cascade processes) and properties of subcritical MHD shear turbulence.

Finally, we would like to discuss the applicability and relevance of our approach to the MRI-driven 3D MHD turbulence in astrophysical disks. Like the MHD shear flow considered here, disk flows are also weakly magnetized [36,42] and hence dominated by shear-induced (transient) effects. Analysis of the dynamics of MRI turbulence in spectral space is important in order to understand its basic nonlinear cascade properties, which play a decisive role in various related processes such as the dependence of turbulence saturation amplitude (turbulent transport) on viscosity and resistivity (in terms of the magnetic Prandtl number [35,51,54,62]), effective turbulent dissipation $[32,45]$, emergence of largescale coherent structures (zonal flows [63-65]), and dynamo action [41,52,66-68]. A spectral analysis of fully developed MRI turbulence in magnetized disks has been carried out in a number of studies $[32,35,41,45]$, as mentioned throughout the text. In these papers, the individual terms in the evolution equation for the kinetic and magnetic spectral energies are examined in wave-number space, as also done here. However, the main focus of these studies was on the dissipative properties of turbulence, which depend on the wave-number magnitude $k$ only, so energy spectra, injection and nonlinear transfer functions angle-averaged in $\mathbf{k}$ space were used to infer the injection wave numbers and cascade directions as well as the dissipation wave numbers. Evidently, such angle-integrated spectral quantities give energy cascade features (direct and inverse) only along the $\mathbf{k}$ direction. But, since one of the main causes of the MRI in disks is shear associated with their differential rotation (see, e.g., [42]), one would expect the dynamics of the resulting turbulence to be essentially anisotropic in $\mathbf{k}$ space (see also Refs. [31,35]), involving nonlinear transverse cascades, similar to those described here, at work. This transverse cascade, arising from the angular dependence of nonlinear spectral transfer functions, is elusive under angle integration and therefore was missing in these studies. To the best of our knowledge, a more complete spectral analysis of MRI-driven turbulence dynamics in 3D Fourier space has not been done yet.

Such a spectral analysis is especially relevant and important for understanding the nature of MRI turbulence in zero net magnetic flux and azimuthal (toroidal) magnetic field configurations, where the linear MRI is manifested as transiently growing nonaxisymmetric modes $[31,38]$; that is, no exponential instability exists in these cases and hence the onset of the MHD turbulence should be subcritical. This subcritical MRI turbulence in disks is currently the subject of active research in the disk community. Although its characteristics in the presence of an imposed nonzero net azimuthal field have been studied extensively (e.g., Refs. [31,33,34]), the main focus was on the effects of viscosity and resistivity on the saturation properties of turbulence, so no clear-cut picture of its basic sustaining mechanism was presented. For the zero net flux case, it is thought that some type of MHD dynamo action must be operative, which generates a large-scale azimuthal field able to sustain the turbulence (e.g., Refs. [41,52,66-68]). The configuration considered here, with a parallel magnetic field, is in fact equivalent to disk flows with an azimuthal background field in the local shearing box model (which, in addition, includes rotation). So, based on this analogy, we speculate that the sustenance mechanism of subcritical MHD shear turbulence presented here can be realized in disk flows too and be responsible for a long-lived MRI turbulence in them. To investigate this in more detail, one should generalize a similar type of spectral analysis of turbulence dynamics in 3D Fourier space in disk flows with nonzero net azimuthal magnetic field in the shearing box approximation.

\section{ACKNOWLEDGMENTS}

We would like to thank Dr. G. Lesur for helping to familiarize us with the specifics of the SNOOPY code and Dr. A. G. Tevzadze for discussions on the physical aspects of the problem. G.R.M. acknowledges financial support from the Rustaveli National Science Foundation.
[1] S. Reddy, P. Schmid, and D. Henningson, SIAM J. Appl. Math. 53, 15 (1993).
[2] L. N. Trefethen, A. E. Trefethen, S. C. Reddy, and T. A. Driscoll, Science 261, 578 (1993). 
[3] P. J. Schmid and D. S. Henningson, Stability and Transition in Shear Flows (Springer, Berlin, 2001).

[4] W. O. Criminale, T. L. Jackson, and R. D. Joslin, Theory and Computation of Hydrodynamic Stability (Cambridge University Press, Cambridge, 2003).

[5] P. J. Schmid, Annu. Rev. Fluid Mech. 39, 129 (2007).

[6] L. H. Gustavsson, J. Fluid Mech. 224, 241 (2006).

[7] B. F. Farrell and P. J. Ioannou, Phys. Fluids 5, 1390 (1993).

[8] S. C. Reddy and D. S. Henningson, J. Fluid Mech. 252, 209 (2006).

[9] B. F. Farrell and P. J. Ioannou, Phys. Fluids 12, 3021 (2000).

[10] T. Gebhardt and S. Grossmann, Phys. Rev. E 50, 3705 (1994).

[11] D. S. Henningson and S. C. Reddy, Phys. Fluids 6, 1396 (1994).

[12] J. S. Baggett, T. A. Driscoll, and L. N. Trefethen, Phys. Fluids 7, 833 (1995).

[13] S. Grossmann, Rev. Mod. Phys. 72, 603 (2000).

[14] S. J. Chapman, J. Fluid Mech. 451, 35 (2002).

[15] B. Eckhardt, T. M. Schneider, B. Hof, and J. Westerweel, Annu. Rev. Fluid Mech. 39, 447 (2007).

[16] W. Horton, J.-H. Kim, G. D. Chagelishvili, J. C. Bowman, and J. G. Lominadze, Phys. Rev. E 81, 066304 (2010).

[17] D. Rempfer, Annu. Rev. Fluid Mech. 35, 229 (2003).

[18] M. E. Stern, Phys. Fluids 6, 636 (1963).

[19] G. I. Ogilvie and J. E. Pringle, Mon. Not. R. Astron. Soc. 279, 152 (1996).

[20] G. D. Chagelishvili, R. G. Chanishvili, J. G. Lominadze, and A. G. Tevzadze, Phys. Plasmas 4, 259 (1997).

[21] P. S. Iroshnikov, Astron. Zh. 40, 742 (1963) [Sov. Astron. 7, 566 (1964)].

[22] R. H. Kraichnan, Phys. Fluids 8, 1385 (1965).

[23] P. Goldreich and S. Sridhar, Astrophys. J. 438, 763 (1995).

[24] S. Boldyrev, Astrophys. J. Lett. 626, L37 (2005).

[25] D. Biskamp, Magnetohydrodynamic Turbulence (Cambridge University Press, Cambridge, 2003).

[26] P. D. Mininni, Annu. Rev. Fluid Mech. 43, 377 (2011).

[27] A. Brandenburg and A. Lazarian, Space Sci. Rev. 178, 163 (2013).

[28] E.-J. Kim, Phys. Rev. Lett. 96, 084504 (2006).

[29] J. Douglas, E.-J. Kim, and A. Thyagaraja, Phys. Plasmas 15, 052301 (2008).

[30] A. P. L. Newton and E.-J. Kim, Phys. Rev. Lett. 102, 165002 (2009).

[31] J. Hawley, C. Gammie, and S. Balbus, Astrophys. J. 440, 742 (1995).

[32] S. Fromang and J. Papaloizou, Astron. Astrophys. 476, 1113 (2007).

[33] X. Guan, C. F. Gammie, J. B. Simon, and B. M. Johnson, Astrophys. J. 694, 1010 (2009).

[34] J. B. Simon and J. F. Hawley, Astrophys. J. 707, 833 (2009).

[35] G. Lesur and P.-Y. Longaretti, Astron. Astrophys. 528, A17 (2011).

[36] P. J. Armitage, Annu. Rev. Astron. Astrophys. 49, 195 (2011).

[37] G. Zimbardo, A. Greco, L. Sorriso-Valvo, S. Perri, Z. Vörös, G. Aburjania, K. Chargazia, and O. Alexandrova, Space Sci. Rev. 156, 89 (2010).

[38] S. A. Balbus and J. F. Hawley, Astrophys. J. 400, 610 (1992).

[39] Z. D. Dimitrov, Y. G. Maneva, T. S. Hristov, and T. M. Mishonov, Phys. Plasmas 18, 082110 (2011).
[40] D. Gogichaishvili, G. Chagelishvili, R. Chanishvili, and J. Lominadze, J. Plasma Phys. (to be published), arXiv:1403.6147 [physics.plasma-ph].

[41] S. W. Davis, J. M. Stone, and M. E. Pessah, Astrophys. J. 713, 52 (2010).

[42] S. A. Balbus, Annu. Rev. Astron. Astrophys. 41, 555 (2003).

[43] G. D. Chagelishvili, R. G. Chanishvili, T. S. Hristov, and J. G. Lominadze, Sov. Phys.-JETP 94, 434 (2002).

[44] A. Alexakis, P. D. Mininni, and A. Pouquet, New J. Phys. 9, 298 (2007).

[45] J. B. Simon, J. F. Hawley, and K. Beckwith, Astrophys. J. 690, 974 (2009).

[46] G. R. Mamatsashvili, G. D. Chagelishvili, G. Bodo, and P. Rossi, Mon. Not. R. Astron. Soc. 435, 2552 (2013).

[47] M. E. Pessah and C.-K. Chan, Astrophys. J. 751, 48 (2012).

[48] The usual Reynolds numbers defined in terms of the half-domain size, $L_{x} / 2$, and the mean flow velocity at the domain boundary, $U_{0, \text { max }}=S L_{x} / 2, \mathrm{Re}^{*}=L_{x} U_{0, \text { max }} / 2 v, \mathrm{Rm}^{*}=L_{x} U_{0, \text { max }} / 2 \eta$, are related to the Reynolds numbers used here by $\mathrm{Re}^{*}=L_{x}^{2} \mathrm{Re} / 4$, $\mathrm{Rm}^{*}=L_{x}^{2} \mathrm{Rm} / 4$. So, for $L_{x}=400$ and $\mathrm{Re}=\mathrm{Rm}=5$, these numbers are actually quite large: $\mathrm{Re}^{*}=\mathrm{Rm}^{*}=2.0 \times 10^{5}$.

[49] The code is available for download at G. Lesur's web page, http://ipag.obs.ujf-grenoble.fr/ /esurg/snoopy.html.

[50] C. Canuto, M. Y. Hussaini, A. Quarteroni, and T. A. Zang, Spectral Methods in Fluid Dynamics (Springer, Berlin, 1988).

[51] G. Lesur and P.-Y. Longaretti, Mon. Not. R. Astron. Soc. 378, 1471 (2007).

[52] G. Lesur and G. I. Ogilvie, Astron. Astrophys. 488, 451 (2008).

[53] G. Lesur and J. C. B. Papaloizou, Astron. Astrophys. 513, A60 (2010).

[54] P.-Y. Longaretti and G. Lesur, Astron. Astrophys. 516, A51 (2010).

[55] E. L. Rempel, G. Lesur, and M. R. E. Proctor, Phys. Rev. Lett. 105, 044501 (2010).

[56] J. Herault, F. Rincon, C. Cossu, G. Lesur, G. I. Ogilvie, and P.-Y. Longaretti, Phys. Rev. E 84, 036321 (2011).

[57] Y. Duguet, P. Schlatter, and D. S. Henningson, J. Fluid Mech. 650, 119 (2010).

[58] Similar anisotropic spectra were also observed in the simulations of MHD turbulence driven by the MRI in the presence of shear [31, 35].

[59] S. Fromang, Astron. Astrophys. 514, L5 (2010).

[60] D. Biskamp and H. Welter, Phys. Fluids B 1, 1964 (1989).

[61] C. C. Wu and T. Chang, J. Atmos. Sol-Terr. Phys. 63, 1447 (2001).

[62] S. Fromang, J. Papaloizou, G. Lesur, and T. Heinemann, Astron. Astrophys. 476, 1123 (2007).

[63] S. Fromang and R. P. Nelson, Mon. Not. R. Astron. Soc. 364, L81 (2005).

[64] A. Johansen, A. Youdin, and H. Klahr, Astrophys. J. 697, 1269 (2009).

[65] J. B. Simon, K. Beckwith, and P. J. Armitage, Mon. Not. R. Astron. Soc. 422, 2685 (2012).

[66] A. Brandenburg, A. Nordlund, R. F. Stein, and U. Torkelsson, Astrophys. J. 446, 741 (1995).

[67] X. Guan and C. F. Gammie, Astrophys. J. 728, 130 (2011).

[68] G. Bodo, F. Cattaneo, A. Mignone, and P. Rossi, Astrophys. J. 761, 116 (2012). 